\title{
Quantum Dressing Orbits on Compact Groups
}

\author{
Branislav Jurčo ${ }^{1, \star}$ and Pavel Šťvíček ${ }^{2}$ \\ 1 A. Sommerfeld Institute, Technical University of Clausthal, \\ W-3392 Clausthal-Zellerfeld, Germany, and Department of Optics, Palacký University, Vídeňská 15, \\ CS-77146 Olomouc, Czechoslovakia $\star \star$ \\ 2 Department of Mathematics, Faculty of Nuclear Science, Trojanova 13, CS-12000 Prague, \\ Czechoslovakia
}

Received January 20, 1992; in revised form July 5, 1992

\begin{abstract}
The quantum double is shown to imply the dressing transformation on quantum compact groups and the quantum Iwasawa decompositon in the general case. Quantum dressing orbits are described explicitly as $*$-algebras. The dual coalgebras consisting of differential operators are related to the quantum Weyl elements. Besides, the differential geometry on a quantum leaf allows a remarkably simple construction of irreducible $*$-representations of the algebras of quantum functions. Representation spaces then consist of analytic functions on classical phase spaces. These representations are also interpreted in the framework of quantization in the spirit of Berezin applied to symplectic leaves on classical compact groups. Convenient "coherent states" are introduced and a correspondence between classical and quantum observables is given.
\end{abstract}

\section{Contents}

0. Introduction . . . . . . . . . . . . . . . . . . . . . . . . . . . . . . . 97

1. Quantum Groups . . . . . . . . . . . . . . . . . . . . 98

2. Poisson Lie Structure on Simple Lie groups . . . . . . . . . . . . . . . . . . 101

3. Quantum Dressing Transformation . . . . . . . . . . . . . . . . . . . . 104

4. Quantum Double for Compact Groups . . . . . . . . . . . . . . . . . . . . 107

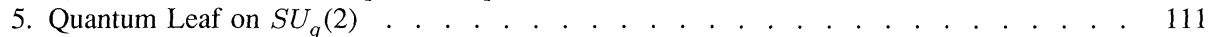

6. Quantum Orbit Related to Quantum Weyl Element . . . . . . . . . . . . 115

7. Irreducible *-Representations for Algebras of Quantum Functions on Compact Groups . 119

8. Quantization of Symplectic Leaves of the Poisson Lie Structure . . . . . . . . . . 123

References . . . . . . . . . . . . . . . . . . 126

\section{Introduction}

Quantum groups were recently introduced by Drinfel'd [7], Jimbo [10], and Woronowicz [30]. In Woronowicz's approach a comnpact quantum group is regarded as a

\footnotetext{
* Humboldt Fellow

** Permanent address
} 
deformation of the $C^{*}$-algebra of continuous functions on a compact group. More generally, a quantum group is understood as a deformation (quantization) of a Poisson Lie group. For this reason we refer to [24] where a nice description of such a deformation in the spirit of the so-called F-R-T (Faddeev-Reshetikhin-Takhtajan) approach to quantum groups [19] is given. Besides, in [21] an interesting attempt has been made to describe $S U_{q}(2)$ as a Rieffel's strict deformation quantization of Poisson Lie group $S U(2)$.

Let us now summarize the structure of the paper as well as the presented results. In Sect. 1, some basic facts about quantum groups are recalled. We follow mainly F-R-T approach [19]. In Sect. 2, we collect some known results needed in the sequel about Poisson Lie structure on compact groups and about the classical dressing transformation. Besides, we want to stress the usually overlooked fact that a complex simple Lie group $G$ also possesses a Poisson Lie structure making the Iwasawa decomposition a Poisson diffeomorphism provided $G$ is considered as a real manifold and the signs on both factors are chosen properly.

Section 3 is devoted to application of the double group construction to quantum compact groups. In analogy with the classical case the quantum double implies the quantum dressing transformation. In Sect. 4 , the quantum double for compact groups is described in terms of $R$-matrices rather than using the representation theory as it was done by Podles' and Woronowicz in [17]. Further we present the general case of the quantum Iwasawa decomposition (the special case of $S L_{q}(2, \mathbb{C})$ was described again in [17]).

The goal of the next two Sects. 5, 6 is to describe explicitly quantum dressing orbits as non-commutative $*$-algebras, the restriction morphism from the quantum group onto the quantum leaf, differential calculus on the orbits and to establish the relation with the quantum Weyl elements defined in $[11,13]$. The differential operators with constant coefficients form the dual coalgebra. The dual of the restriction morphism is described explicitly. Consequently, the quantum Weyl element can be regarded as the base point of the quantum dressing orbit in analogy with the classical case.

In Sect.7, we first recall some basic facts from the theory of irreducible *-representations of compact quantum groups. After that, we present a construction of these representations realized in a space of holomorphic functions living on the classical Poisson leaf. Its remarkable feature is that it is related in a simple and clear manner to the differential geometry of the quantum leaf. In Sect. 8 we want to stress a point of view according to which the above described representations realized in spaces of holomorphic functions can be also understood in the framework of quantization methods. Besides, we introduce convenient coherent states and discuss the correspondence principle.

\section{Quantum Groups}

In the F-R-T approach the basic object through which the simple quantum groups (more precisely algebras of functions on the simple $q$-groups) of types $A_{n-1}, B_{n}$, $C_{n}$, and $D_{n}$ are introduced is the $R$-matrix satisfying the Yang-Baxter equation

$$
R_{12} R_{13} R_{23}=R_{23} R_{13} R_{12}
$$


where $R$ is an $N^{2} \times N^{2}$ matrix ( $N=n$ in the case $A_{n-1}, N=2 n$ in the cases $C_{n}$, $D_{n}, N=2 n+1$ in the case $B_{n}$ ). In the case of $A_{n-1}$ we have

$$
q^{1 / n} R=q \sum_{i=1}^{n} e_{\imath \imath} \otimes e_{\imath i}+\sum_{\substack{\imath, \jmath=1 \\ i \neq j}}^{n} e_{i \imath} \otimes e_{\jmath \jmath}+\left(q-q^{-1}\right) \sum_{\substack{\imath, \jmath=1 \\ i>j}}^{n} e_{\imath j} \otimes e_{j i}
$$

where $q \in \mathbb{C} \backslash\{0\}$ and $e_{i j}$ are $n \times n$ matrix units.

In the remaining cases

$$
\begin{aligned}
R= & q \sum_{\substack{i=1 \\
\imath \neq i^{\prime}}}^{N} e_{\imath \imath} \otimes e_{\imath i}+e_{\frac{N+1}{2}, \frac{N+1}{2}} \otimes e_{\frac{N+1}{2}, \frac{N+1}{2}}+\sum_{\substack{i, \jmath=1 \\
\imath \neq j, j^{\prime}}}^{N} e_{i i} \otimes e_{j j} \\
& +q^{-1} \sum_{\substack{i=1 \\
i \neq \imath^{\prime}}}^{N} e_{i^{\prime} \imath^{\prime}} \otimes e_{i i}+\left(q-q^{-1}\right) \sum_{\substack{i, j=1 \\
i>j}}^{N} e_{i j} \otimes e_{j i} \\
& +\left(q-q^{-1}\right) \sum_{\substack{i, j=1 \\
i>j}}^{N} q^{\varrho_{\imath}-\varrho_{\jmath} \varepsilon_{\imath} \varepsilon_{\jmath} e_{i j} \otimes e_{i^{\prime} j^{\prime}}, \quad q \in \mathbb{C} \backslash\{0\} ;}
\end{aligned}
$$

the second term occurs in the $B_{n}$ case only, $i^{\prime}=N+1-i, \varepsilon_{i}=1, i=1, \ldots, N$, for the cases $B_{n}$ and $D_{n}, \varepsilon_{i}=1, i=1, \ldots, \frac{N}{2}, \varepsilon_{\imath}=-1, i=\frac{N}{2}+1, \ldots, N$, for the case $C_{n}$ and

$$
\left(\varrho_{1}, \ldots, \varrho_{N}\right)= \begin{cases}(n-1 / 2, n-3 / 2, \ldots, 1 / 2,0,-1 / 2, \ldots,-n+1 / 2) & \text { for } B_{n} \\ (n, n-1, \ldots, 1,-1, \ldots,-n) & \text { for } C_{n} . \\ (n-1, n-2, \ldots, 1,0,0,-1, \ldots,-n+1) & \text { for } D_{n}\end{cases}
$$

All the matrices $R$ are unimodular.

The algebra of holomorphic functions on the $S L_{q}(n)$ group is then defined as the factor algebra of the free associative unital $\mathbb{C}$-algebra $\mathbb{C}\left\langle t_{r j}\right\rangle(i, j=1, \ldots, n)$ by the following relations:

$$
\begin{gathered}
R T_{1} T_{2}=T_{2} T_{1} R \\
\operatorname{det}_{q} T=\sum_{\sigma \in S_{n}}(-q)^{2(\sigma)} t_{1 \sigma_{1}} t_{2 \sigma_{2}} \ldots t_{n \sigma_{n}}=1 .
\end{gathered}
$$

Here $\operatorname{det}_{q} T$ denotes the quantum determinant, $T=\left(t_{\imath, j}\right)_{l, j=1}^{n}, T_{1}=T \otimes I, T_{2}=I \otimes T$ and $l(\sigma)$ is the number of transpositions in permutation $\sigma . R$ is given by (1.2) in this case. This algebra will be denoted by $\mathscr{C}_{q}^{\text {hol }}(S L(n))$ and it is a Hopf algebra with comultiplication

$$
\Delta\left(t_{i j}\right)=t_{i k} \otimes t_{k j}, \quad \Delta 1=1 \otimes 1
$$

counit

$$
e\left(t_{i j}\right)=\delta_{i j}, \quad e(1)=1
$$


and antipode

$$
S\left(t_{j \imath}\right)=(-q)^{\jmath-\imath} \sum_{\sigma \in S}(-q)^{l(\sigma)} t_{1 \sigma_{1}} \ldots t_{i-1 \sigma_{i-1}} t_{\imath+1 \sigma_{\imath+1}} \ldots t_{n \sigma_{n}},
$$

where $\sigma=\left(\sigma_{1}, \ldots, \sigma_{i-1}, \sigma_{\imath+1}, \ldots, \sigma_{n}\right)=\sigma(1, \ldots, j-1, j+1, \ldots, n)$.

Algebra of quantum functions $\mathscr{C}_{q}^{\text {hol }}(G)(G=S O(N), S p(N))$ is defined as the factor algebra of free associative unital $\mathbb{C}$-algebra $\mathbb{C}\left\langle t_{\imath j}\right\rangle(i, j=1, \ldots, N)$ by relations (1.4) involving the corresponding $R$-matrix (1.3) and relations

$$
T C T^{t} C^{-1}=C T^{t} C^{-1} T=I ;
$$

here $t$ denotes the transposition and $C$ is an $\mathbb{C}$-number matrix given by $C=C_{0} q^{Q}$, with $\varrho=\left(\varrho_{1}, \ldots, \varrho_{N}\right)$ and $\left(C_{0}\right)_{i j}=\varepsilon_{\imath} \delta_{i^{\prime} j}$. These algebras are Hopf algebras with comultiplication (1.6), counit (1.7) and antipode

$$
S(T)=C T^{t} C^{-1}
$$

For $q \in \mathbb{R}$, the $R$-matrices are real and fulfill

$$
R_{12}^{*}=R_{21}
$$

Any of the described algebras $\operatorname{Ag}_{q}^{\text {hol }}(G)$ admit the following $*$-structure

$$
t_{i j}^{*}=S\left(t_{\jmath \imath}\right) \text {. }
$$

The star operation is an algebra antihomomorphism, coalgebra homomorphism and involution. The resulting *-Hopf algebra is called a compact form (compact matrix pseudogroup of [30]) and will be denoted by $\mathscr{H}_{q}(K)$, where $K \subset G$ is the maximal compact subgroup. In this case we shall use the symbol $U$ for the matrix of generators instead of $T$. This $U$ satisfies (1.4) and, moreover,

$$
S(U)=U^{*}
$$

The dual Hopf algebras to the above mentioned quantum groups are introduced following $[7,10,19]$. Let $\mathfrak{g}$ be a simple Lie algebra, $\alpha_{1}, \ldots, \alpha_{r}$ its simple roots and $A_{i j}=2\left(\alpha_{i}, \alpha_{j}\right) /\left(\alpha_{j}, \alpha_{j}\right)$ its Cartan matrix. Algebra $U_{h} \mathfrak{g}, h \in \mathbb{C}$, is a $\mathbb{C}[[h]]$ algebra with generators $H_{\imath}, X_{i}^{ \pm}, i=1, \ldots, r$, and relations

$$
\begin{gathered}
{\left[H_{i}, H_{j}\right]=0, \quad\left[H_{i}, X_{j}^{ \pm}\right]= \pm\left(\alpha_{\imath}, \alpha_{j}\right) X_{j}^{ \pm}, \quad\left[X_{i}^{+}, X_{\jmath}^{-}\right]=\delta_{\imath \jmath} \frac{\operatorname{sh}\left(h H_{i}\right)}{\operatorname{sh}(h)},} \\
\sum_{k=0}^{m}(-1)^{k}\left[\begin{array}{c}
m \\
k
\end{array}\right]_{q_{\imath}}\left(X_{i}^{ \pm}\right)^{k} X_{j}^{ \pm}\left(X_{i}^{ \pm}\right)^{m-k}=0
\end{gathered}
$$

for $i \neq j$, where $m=1-A_{i j}, q_{i}=e^{-h\left(\alpha_{i}, \alpha_{\imath}\right) / 2}$ and

$$
[k]_{q}=\frac{q^{k}-q^{-k}}{q-q^{-1}} .
$$

The quantum factorials and the quantum binomial coefficients are then introduced as usual. The subscript $q$ will be omitted if not necessary. The normalization is chosen in such a way that $\left(\alpha_{i}, \alpha_{i}\right)=2$ for the short roots. 
Algebra $U_{h} \mathfrak{g}$ is a Hopf algebra with comultiplication

$$
\begin{gathered}
\Delta\left(H_{i}\right)=H_{i} \otimes 1+1 \otimes H_{i}, \\
\Delta\left(X_{\imath}^{ \pm}\right)=X_{i}^{ \pm} \otimes \exp \left(h H_{i} / 2\right)+\exp \left(-h H_{i} / 2\right) \otimes X_{i}^{ \pm}
\end{gathered}
$$

and antipode $S$

$$
S\left(H_{i}\right)=-H_{i}, \quad S\left(X_{i}^{ \pm}\right)=-e^{h \delta} X_{i}^{ \pm} e^{-h \delta},
$$

where $\delta$ is the element of the Cartan subalgebra corresponding to the half-sum of positive roots. For $h \in \mathbb{R}$, the Hopf algebra $U_{h} \mathfrak{g}$ admits the following $*$-structure

$$
\left(H_{i}\right)^{*}=H_{i}, \quad\left(X_{i}^{ \pm}\right)^{*}=X_{i}^{\mp} .
$$

The resulting $*$-Hopf algebra can be also regarded as a deformation of the complexified eneveloping algebra $U(\mathfrak{k})$ where $\mathfrak{k} \subset \mathfrak{g}$ is the maximal compact subalgebra.

Without loss of generality we may restrict the values of $q \in(0,1)$. Letting $q=e^{-h}$ we have a pairing between $U_{h} \mathfrak{g}$ and $\mathscr{b}_{q}(K)$ determined by the rules

$$
\begin{gathered}
\langle x y, c\rangle=\langle x \otimes y, \Delta(c)\rangle, \\
\langle x, c d\rangle=\langle\Delta(x), c \otimes d\rangle, \\
\left\langle x^{*}, c\right\rangle=\left\langle x,(S c)^{*}\right\rangle^{*},
\end{gathered}
$$

and by the requirement that $\left\langle H_{i}, U\right\rangle,\left\langle X_{\imath}^{ \pm}, U\right\rangle$ coincide with the corresponding matrices in the fundamental (vector) representation of the classical Lie algebra $\mathfrak{g}$. Using the "restriction" homomorphism

$$
p_{K}: \mathscr{f}_{q}^{\mathrm{hol}}(G) \rightarrow \mathscr{f}_{q}(K),
$$

between the Hopf algebras which is determined by $p_{k}(T)=U$ we have also a pairing between $U_{h} \mathfrak{g}$ and $\mathscr{A}_{q}^{\mathrm{hol}}(G)$.

Let $\check{\varphi}_{i, h}: U_{h} \operatorname{sl}(2) \rightarrow U_{h} \mathfrak{g}$ denote the canonical embedding of $\operatorname{sl}(2)$-triple corresponding to the $i^{\text {th }}$ simple root. It is not hard to see that there are corresponding embeddings of quantum groups $\varphi_{\imath, h}: S U_{q_{2}}(2) \rightarrow K_{q}$, with $q_{i}=q$ for all simple roots in the case of $A_{n-1}$ and $D_{n} ; q_{i}=q$, for $i=1, \ldots, n-1$ and $q_{n}=q^{2}$ in the $C_{n}$ case and $q_{i}=q$, for $i=1, \ldots, n-1$ and $q_{n}=q^{1 / 2}$ in the $B_{n}$ case. This means that we have a morphism of $*$-Hopf algebras

$$
\varphi_{i, h}: \mathscr{A}_{q}(K) \rightarrow \mathscr{C}_{q_{i}}(S U(2))
$$

\section{Poisson Lie Structure on Simple Lie Groups}

There is a wide literature about this topic going back to Drinfeld's original treatment [7] (we refer the interested reader for further details e.g. to $[14,20]$ ). In the context 
of the present paper, an important point of view is that one strengthening the role of the Iwasawa decomposition [14]. Let

$$
\begin{gathered}
G=K \times A N, \\
\mathfrak{g}_{\mathbb{R}}=\mathfrak{k} \oplus \mathfrak{a n},
\end{gathered}
$$

be the Iwasawa decomposition of a connected simple complex Lie group and of its Lie algebra $\mathfrak{g}$ considered over reals, respectively. As usual, $K$ is the maximal compact subgroup and $A N$ is the solvable subgroup in $G$. Having equipped $\mathfrak{g}_{\mathbb{R}}$ with the form $\langle\cdot, \cdot\rangle=\operatorname{Im} B(\cdot, \cdot)$, where $B$ is the Killing form on the complex algebra $\mathfrak{g}$, one gets a Manin triple $\left(\mathfrak{g}_{\mathbb{R}}, \mathfrak{k}, \mathfrak{a n}\right)$. The subalgebras $\mathfrak{k}$ and $\mathfrak{a n}$ induce one on the other one-cocycles

$$
\mu_{K}: \mathfrak{k} \rightarrow \mathfrak{k} \wedge \mathfrak{k}, \quad \mu_{A N}: \mathfrak{a n} \rightarrow \mathfrak{a n} \wedge \mathfrak{a n} .
$$

On the other hand, these cocycles determine Poisson Lie structure on the groups $K$ and $A N$, respectively. But according to the following discussion, if we want to turn $G$ also into a Poisson Lie group and the Iwasawa decomposition into a Poisson diffeomorphism we have to take $\mu_{A N}$ with the opposite sign and consequently to change the sign at the Poisson bracket on $A N$.

We define a classical $r$-matrix $\tilde{r} \in \mathfrak{g}_{\mathbb{R}} \wedge \mathfrak{g}_{\mathbb{R}}$ by

$$
\tilde{r}=\frac{1}{2} \sum Y_{s} \wedge Z_{s}
$$

where $\left\{Y_{s}\right\}$ and $\left\{Z_{s}\right\}$ are dual basis in an and $\mathfrak{k}$, respectively. The corresponding one-cocycle $\mu$ on $\mathfrak{g}_{\mathbb{R}}$,

$$
\mu(X)=-\operatorname{ad}(X) \tilde{r},
$$

induces a Lie bracket on $\mathfrak{g}_{\mathbb{R}}^{*}$ and a Poisson Lie structure on $G$. Having identified $\mathfrak{g}_{\mathbb{R}}^{*}$ with $\mathfrak{g}_{\mathbb{R}}$ using the form $\langle.,$.$\rangle we have$

$$
\mathfrak{g}_{\mathbb{R}}^{*}=\mathfrak{k}^{-} \oplus \mathfrak{a n}
$$

as a direct sum of Lie algebras. The symbol $\mathfrak{k}^{-}$means that the Lie bracket in $\mathfrak{k}$ is taken with the opposite sign. One can check without problems that

$$
\mu \mid \mathfrak{k}=\mu_{K} \quad \text { and } \quad \mu \mid \mathfrak{a n}=-\mu_{A N} .
$$

An immediate consequence of $(2.5)$ is

Proposition 3.1. The Iwasawa decomposition (2.1a) is a Poisson mapping.

Proof. It is not difficult to verify that a sufficient and necessary condition for a subgroup $H \subset G$ to be a Poisson subgroup is

$$
\mu(\mathfrak{h}) \subset \mathfrak{h} \wedge \mathfrak{h}
$$

(this criterion is different from that one given in $[20,14]$ ). Hence $K$ and $A N$ are Poisson subgroups in $G, K \times A N$ is a Poisson subgroup in $G \times G$. As the multiplication $G \times G \rightarrow G$ is a Poisson mapping the same is true for the diffeomorphism $K \times A N \rightarrow G$. Q.E.D.

Let us now compute the Poisson bracket on $G$ explicitly. Generally it holds: if some $r$-matrix is expressed as

$$
r=\sum_{i<j} r^{i j} X_{i} \wedge X_{j}
$$


and $\xi_{\imath}^{R}\left(\xi_{i}^{L}\right)$ is the right-(left-)invariant vector field on $G$ corresponding to $X_{i}$ then

$$
\{f, g\}=\sum_{i<\jmath} r^{i j}\left(\xi_{i}^{R} \cdot f \xi_{j}^{R} \cdot g-\xi_{i}^{L} \cdot f \xi_{\jmath}^{L} \cdot g-(f \leftrightarrow g)\right) .
$$

It follows that if $\tau$ is a complex representation of $\mathfrak{g}$ and $T$ the corresponding holomorphic representation of $G$ then

$$
\begin{gathered}
\{T \underset{,}{\otimes} T\}=[(\tau \otimes \tau) r, T \otimes T], \\
\left\{T^{*}{\underset{,}{\otimes}\}}^{*}=T_{1}^{*}\left(\tau^{*} \otimes \tau\right) r T_{2}-T_{2}\left(\tau^{*} \otimes \tau\right) r T_{1}^{*} .\right.
\end{gathered}
$$

In our case, we are able to express $\tilde{r}$ using the Weyl generators in $\mathfrak{g}\left(B\left(X_{\alpha}, X_{-\alpha}\right)=1\right.$, $\left.\left[X_{\alpha}, X_{-\alpha}\right]=H_{\alpha}\right)$

$$
\tilde{r}=\sum_{\alpha>0}\left(\frac{1}{2} X_{\alpha} \wedge i X_{-\alpha}+\frac{1}{2} i X_{\alpha} \wedge X_{-\alpha}+X_{\alpha} \wedge i X_{\alpha}+H_{\alpha} \wedge i H_{\alpha}\right)
$$

Note that the symbols $i X_{\alpha}, i H_{\alpha}$ etc. are rigid in $\mathfrak{g}_{\mathbb{R}}, i=(-1)^{1 / 2}$. Taking into account that the element

$$
\sum_{\alpha>0}\left(X_{\alpha} \otimes X_{-\alpha}+X_{-\alpha} \otimes X_{\alpha}+2 H_{\alpha} \otimes H_{\alpha}\right)
$$

is $\operatorname{ad}(\mathfrak{g})$-invariant we obtain finally

$$
\begin{gathered}
\{T \otimes T\}=[(\tau \otimes \tau) r, T \otimes T], \\
\left\{T^{*} \underset{,}{\otimes}\right\}=-T_{1}^{*}(\tau \otimes \tau) r T_{2}+T_{2}(\tau \otimes \tau) r T_{1}^{*},
\end{gathered}
$$

where

$$
r=-2 i \sum_{\alpha>0}\left(X_{-\alpha} \otimes X_{\alpha}+H_{\alpha} \otimes H_{\alpha}\right) \in \mathfrak{g} \otimes \mathfrak{g} .
$$

It is useful to compare these relations with the correspondence rules relating the classical case with the quantum one:

$$
\begin{gathered}
\Delta(Z)=Z \otimes 1+1 \otimes Z+i \frac{1}{2} h \mu(Z)+O\left(h^{2}\right), \\
f g-g f=i h\{f, g\}+O\left(h^{2}\right), \\
R=I-i h r+O\left(h^{2}\right) .
\end{gathered}
$$

The complex unit is here necessary to guarantee reality of the Poisson bracket. In fact, the $r$-matrix (2.13) should be multiplied by some positive constant to meet the rules (2.14). This is related with renormalization of the Killing form on $\mathfrak{g}$. The relation (2.11) corresponds to (1.4), the quantum counterpart to (2.12) will be discussed later (4.7b).

Because the projections

$$
\Pi_{K}: G \rightarrow K \quad \text { and } \quad \Pi_{A N}: G \rightarrow A N
$$

induced by the Iwasawa decomposition are Poisson mappings the below defined left action of $A N$ on $K$ and the right action of $K$ on $A N$ possess the same property:

$$
\begin{aligned}
L: A N \times K \rightarrow K, & L(u, g)=\Pi_{K}(u g), \\
R: A N \times K \rightarrow A N, & R(u, g)=\Pi_{A N}(u g) .
\end{aligned}
$$


These actions are called dressing transformations. The following "twisted multiplication" rule is clearly valid

$$
L\left(u, g_{1} g_{2}\right)=L\left(u, g_{1}\right) L\left(R\left(u, g_{1}\right), g_{2}\right) .
$$

According to $[14,23]$, every symplectic leaf of the Poisson structure on $K$ has a form $X_{W} \cdot t$, where $t$ is an element of the maximal torus $M$ in $K$ and $X_{W}$ is the Schubert cell corresponding to some element $W$ of the Weyl group. In fact, $X_{W}$ coincides with the orbit in the dressing action containing $W$. Perhaps this is the most distinguishing property of the dressing transformation. Let now $W=W_{i_{1}} \ldots W_{\imath_{k}}$ be the reduced decomposition of $W$, where $W_{i}$ is the Weyl element of the $\operatorname{sl}(2)$ triple corresponding to the $i^{\text {th }}$ simple root. It is known that every symplectic leaf $X_{W}$ decomposes as a direct product of symplectic leaves $X_{W_{\imath}}$,

$$
X_{W}=X_{W_{\imath_{1}}} \ldots X_{W_{\imath_{k}}}
$$

and that two different reduced decompositions of the same Weyl element give in the above decomposition isomorphic symplectic manifolds.

Now we give a more detailed description of the symplectic manifolds $X_{W_{i}}$. Let us begin with the simplest case of $S U(2)$ Lie group. As is well known [28] in this case this symplectic leaf is of the form

$$
X=\left\{\left(\begin{array}{cc}
(1+\bar{z} z)^{-1 / 2} z & (1+\bar{z} z)^{-1 / 2} \\
-(1+\bar{z} z)^{-1 / 2} & (1+\bar{z} z)^{-1 / 2} \bar{z}
\end{array}\right) \in S U(2) ; z \in \mathbb{C}\right\}
$$

and the Poisson bracket on this symplectic leaf is given by

$$
\{z, \bar{z}\}=2 i(1+\bar{z} z) .
$$

The dressing transformation on the orbit $X$ can be written explicitly,

$$
L(u, z)=\alpha^{2} z-\beta \alpha,
$$

where $u=\left(\begin{array}{cc}\alpha & \beta \\ 0 & \alpha^{-1}\end{array}\right) \in A N$.

In the general case [22] the symplectic leaf (2.18) can be described as a direct product of $k$ copies of the symplectic manifold $X$ with $k$ pairs of coordinates $\left(z_{1}, \bar{z}_{1}, \ldots, z_{k}, \bar{z}_{k}\right)$, where $z_{j}$ is the coordinate on $X_{W_{j}}$, and with Poisson brackets

$$
\left\{z_{j}, \bar{z}_{k}\right\}=i \alpha_{\jmath}\left(1+\bar{z}_{j} z_{j}\right) \delta_{j k}
$$

where $\alpha_{\jmath}=2$ for all $j$ in the cases $A_{n-1}$ and $D_{n}, \alpha_{j}=2$ for $W_{i_{j}}$ corresponding to the first $n-1$ roots in the cases $B_{n}$ and $C_{n}$ and $\alpha_{j}=1$ for the $n^{\text {th }}$ root in the case of $B_{n}$ and $\alpha_{\jmath}=4$ for the $n^{\text {th }}$ root in the case $C_{n}$. If $\varphi_{\imath}$ denotes the group embedding corresponding to the $i^{\text {th }}$ simple root then we can write in matrix form

$$
X_{W}=\varphi_{\imath_{1}}(X) \ldots \varphi_{i_{k}}(X),
$$

where we assume that in the $i^{\text {th }}$ term in the product $z_{\imath}$ stands instead of $z$.

\section{Quantum Dressing Transformation}

The basic notion we start from in this section is the quantum double. Its definition goes back to Drinfel'd [7] and it was widely applied to quantum enveloping algebras 
[19]. An extensive study of related problems was given in [18]. Let us start from the definition. Let $\mathscr{A}_{c}$ be a $*$-Hopf algebra and $\langle\cdot, \cdot\rangle: \mathscr{A}_{d} \otimes \mathscr{A}_{c} \rightarrow \mathbb{C}$ be the pairing between $\mathscr{b}_{c}$ and the dual vector space $\mathscr{t}_{d} \cdot \mathscr{b}_{d}$ is also equipped with a structure of *-Hopf algebra following unambiguously from the rules

$$
\begin{gathered}
\langle u v, c\rangle=\langle u \otimes v, \Delta(c)\rangle, \\
\langle\Delta(u), c \otimes d\rangle=\langle u, d c\rangle, \\
\langle S u, c\rangle=\left\langle u, S^{-1} c\right\rangle, \\
\varepsilon(u)=\langle u, 1\rangle, \\
\langle 1, c\rangle=\varepsilon(c), \\
\left\langle u^{*}, c\right\rangle=\left\langle u,(S a)^{*}\right\rangle^{*} .
\end{gathered}
$$

The $*$-algebra $\mathscr{D}=\mathscr{A}_{c} \otimes \mathscr{A}_{d}$ can be turned into a $*$-Hopf algebra as follows. Let

$$
\varrho=\sum x_{s} \otimes a_{s}
$$

be the canonical element in $\mathscr{C}_{d} \otimes \mathscr{C}_{c}$ with $\left\{x_{s}\right\}$ and $\left\{a_{s}\right\}$ being dual basis in $\mathscr{C}_{d}$ and $\mathscr{A}_{c}$, respectively. Utilizing the mapping

$$
\Phi: \mathscr{A}_{c} \otimes \mathscr{A}_{d} \rightarrow \mathscr{A}_{d} \otimes \mathscr{A}_{c}, \quad \Phi(c \otimes u)=\varrho(u \otimes c) \varrho^{*},
$$

one can define

$$
\begin{gathered}
\Delta=(\mathrm{id} \otimes \Phi \otimes \mathrm{id})\left(\Delta_{c} \otimes \Delta_{d}\right), \\
\varepsilon=\varepsilon_{c} \otimes \varepsilon_{d}, \\
S=P_{12}\left(S_{d} \otimes S_{c}\right) \Phi .
\end{gathered}
$$

Here and everywhere in what follows $P_{i j}$ designates the permutation (flip) mapping between the indicated factors in some tensor product.

The canonical element $\varrho$ is known to have properties [17]

$$
\begin{gathered}
\varrho^{*} \varrho=\varrho \varrho^{*}=1, \\
\left(\text { id } \otimes S_{c}\right) \varrho=\varrho^{*}, \quad\left(S_{d} \otimes \text { id }\right) \varrho^{*}=\varrho,
\end{gathered}
$$

and the mapping $\Phi$ fulfills the following important identities:

$$
\begin{gathered}
\left(\varepsilon_{d} \otimes \mathrm{id}\right) \Phi=\mathrm{id} \otimes \varepsilon_{d}, \quad\left(\mathrm{id} \otimes \varepsilon_{c}\right) \Phi=\varepsilon_{c} \otimes \mathrm{id} \\
\quad(\mathrm{id} \otimes \Phi)(\Phi \otimes \mathrm{id})\left(\mathrm{id} \otimes \Delta_{d}\right)=\left(\Delta_{d} \otimes \mathrm{id}\right) \Phi \\
(\Phi \otimes \mathrm{id})(\mathrm{id} \otimes \Phi)\left(\Delta_{c} \otimes \mathrm{id}\right)=\left(\mathrm{id} \otimes \Delta_{c}\right) \Phi .
\end{gathered}
$$

Besides, one can define comultiplication also on $\mathscr{D}^{\prime}=\mathscr{A}_{d} \otimes \mathscr{A}_{c}$ getting thus another *-Hopf algebra,

$$
\Delta^{\prime}=\left(\mathrm{id} \otimes \Phi^{-1} \otimes \mathrm{id}\right)\left(\Delta_{d} \otimes \Delta_{c}\right) .
$$

The mapping $\Phi$ is then an isomorphism of $*$-Hopf algebras. Let us denote by

$$
\iota_{c}: \mathscr{A}_{c} \rightarrow \mathscr{D}, \quad \iota_{d}: \mathscr{A}_{d} \rightarrow \mathscr{D}
$$

the natural embeddings and by

$$
\begin{aligned}
& p_{c}=\mathrm{id} \otimes \varepsilon_{d}: \mathscr{D} \rightarrow \mathscr{A}_{c}, \\
& p_{d}=\varepsilon_{c} \otimes \mathrm{id}: \mathscr{D} \rightarrow \mathscr{A}_{d},
\end{aligned}
$$


the projections. The mappings $\iota_{c}, \iota_{d}$ are $*$-algebra morphisms and $p_{c}, p_{d}$ are $*$-Hopf algebra morphisms. It holds

$$
\left(p_{c} \otimes p_{d}\right) \Delta=\mathrm{id}, \quad\left(p_{d} \otimes p_{c}\right) \Delta=\Phi .
$$

It is useful to observe that after the natural embedding of $\mathscr{A}_{c} \otimes \cdot \mathscr{A}_{d}$ into $\operatorname{Lin}\left(\mathscr{A}_{c}\right)-$ the space of linear operators on $\mathscr{A}_{c}$ - the $*$-Hopf algebra structure can be extended to $\operatorname{Lin}\left(\mathscr{C}_{c}\right)$. This enables one to avoid the difficulties with infinite series. The $*$-algebra structure is defined by

$$
\begin{aligned}
m(A, B) & =m_{c}(A \otimes B) \Delta_{c}, \\
A^{*} c & =\left(A(S c)^{*}\right)^{*} .
\end{aligned}
$$

To define the comultiplication embed $\operatorname{Lin}\left(\mathscr{A}_{c}\right) \otimes \operatorname{Lin}\left(\mathscr{A}_{c}\right)$ into $\operatorname{Lin}\left(\mathscr{A}_{c} \otimes \mathscr{C}_{c}\right)$. Then

$$
\begin{gathered}
\Delta A(1 \otimes c)=\Delta_{c}(A c), \\
\Delta A(c \otimes 1)=\sum\left(1 \otimes c_{k}^{1}\right) \Delta_{c}\left(A c_{k}^{2}\right)\left(1 \otimes S c_{k}^{3}\right),
\end{gathered}
$$

where

$$
\left(\mathrm{id} \otimes \Delta_{c}\right) \Delta_{c} c=\sum c_{k}^{1} \otimes c_{k}^{2} \otimes c_{k}^{3}
$$

The left dressing transformation of $\mathscr{A}_{d}$ on $\mathscr{A}_{c}$ should be a $*$-algebra morphism

$$
L: \mathscr{A}_{c} \rightarrow \mathscr{A}_{d} \otimes \mathscr{C}_{c}
$$

with some additional properties. The notion of quantum left (right) action has now stabilized in the literature [1]. The morphism $L$ is required to satisfy

$$
\begin{gathered}
\left(\varepsilon_{d} \otimes \mathrm{id}\right) L=\mathrm{id}, \\
(\mathrm{id} \otimes L) L=\left(\Delta_{d} \otimes \mathrm{id}\right) L .
\end{gathered}
$$

An important property of the classical dressing transformation is that it preserves Poisson manifolds. This condition is reflected in the quantum case as follows. We say that a two sided ideal $\mathscr{T}$ in $\mathscr{A}_{c}$ is $L$-invariant if

$$
L(\mathscr{T}) \subset \mathscr{A}_{d} \otimes \mathscr{T} .
$$

After identification of $\mathscr{t}_{d} \otimes\left(\mathscr{A}_{c} / \mathscr{T}\right)$ with $\mathscr{t}_{d} \otimes \mathscr{b}_{c} / \mathscr{A}_{d} \otimes \mathscr{T}$ we have the factor action

$$
L_{\mathscr{T}}: \mathscr{A}_{c} / \mathscr{T} \rightarrow \cdot \mathscr{t}_{d} \otimes\left(\mathscr{A}_{c} / \mathscr{T}\right)
$$

Analogy with the classical case suggests the following definition of quantum dressing transformation

$$
L=\Phi \iota_{c}, \quad \text { i.e., } \quad L(c)=\varrho(1 \otimes c) \varrho^{*} .
$$

The following proposition is an immediate consequence of the identities (3.9), (3.10).

Proposition 3.1. The morphism $L$ is a left quantum action. Every two-sided ideal $\mathscr{T} \subset \mathscr{t}_{c}$ is L-invariant.

One can consider as well the dual action. Denoting by $U_{c}$ and $U_{d}$ the $*$-Hopf algebras dual to $\mathscr{t}_{c}$ and $\mathscr{b}_{d}$, respectively, we have the dual morphism

$$
\check{L}: U_{d} \otimes U_{c} \rightarrow U_{c} .
$$


The conditions (3.17), (3.18) are then rewritten

$$
\begin{gathered}
\check{L}(1 \otimes Z)=Z, \\
\check{L}(\xi \otimes \check{L}(\eta \otimes Z))=\check{L}(\xi \eta \otimes Z) .
\end{gathered}
$$

One can introduce analogously the right dressing transformation of $b_{c}$ on $\mathscr{l}_{d}$ as a morphism

$$
R:=\Phi \iota_{d}: /_{d} \rightarrow \cdot /_{d} \otimes \cdot l_{c} .
$$

Note that the mapping $\Phi$ can be rewritten as

$$
\Phi=\left(m_{d} \otimes m_{c}\right) P_{23}(L \otimes R) .
$$

Using this relation and the identity (3.11) one can verify a quantum analogy of (2.17),

$$
\left(\mathrm{id} \otimes \Delta_{c}\right) L=\left(\left(m_{d} \otimes m_{c}\right) P_{23} \otimes \mathrm{id}\right)(L \otimes(R \otimes \mathrm{id}) L) \Delta_{c} .
$$

Its dual version is

$$
\breve{L}\left(\mathrm{id} \otimes m_{c}\right)=m_{c}(\breve{L} \otimes \breve{L}(\breve{R} \otimes \mathrm{id}))\left(P_{23}\left(\Delta_{d} \otimes \Delta_{c}\right) \otimes \mathrm{id}\right) .
$$

Here we have not introduced special symbols for multiplication and comultiplication in the dual Hopf algebra.

It is worth emphasizing that the requirement on the classical dressing transformation is stronger, namely the symplectic leaves should coincide with the dressing orbits. The quantum analogy is more speculative in the present moment and we shall discuss it shortly in Sect. 6 [after the relation (6.21)].

\section{Quantum Double for Compact Groups}

Let us now apply this general construction to quantum compact groups, $\mathscr{L}_{c}=$ . $l_{q}(K)$. The dual quantum group was described in [17] on the base of the representation theory. Here we prefer the approach exploiting existence of the quantum $R$-matrix. Let $U$ be the vector representation of $K_{q}$. The vector representation $\Lambda$ of the dual group $A N_{q}$ contains generators of the algebra. $\iota_{d}=l_{q}(A N)$ as its entries and is determined by the relations

$$
\begin{aligned}
& \left\langle\Lambda_{1} ; U_{2}\right\rangle=R_{21}^{-1}, \\
& \left\langle\Lambda_{1}^{*} ; U_{2}\right\rangle=R_{12}^{-1},
\end{aligned}
$$

In this way the pairing between. $/_{q}(A N)$ and $/_{q}(K)$ is determined unambioguously. For example, it holds: if $\left(j_{1}, \ldots, j_{m}, k_{1}, \ldots, k_{n}\right)$ is any permutation of $(1,2, \ldots, N)$, $N=m+n$, then

$$
\left\langle\Lambda_{\jmath_{1}} \ldots \Lambda_{j_{m}} ; U_{k_{1}} \ldots U_{k_{n}}\right\rangle=\prod R_{k_{\sigma} j_{\nu}}^{-1} .
$$

The product on the RHS is ordered: $R_{k_{\sigma} j_{\nu}}$ stands left to $R_{k_{\sigma^{\prime} \nu_{\nu^{\prime}}}}$ whenever $\sigma=\sigma^{\prime}$, $\nu<\nu^{\prime}$ or $\sigma>\sigma^{\prime}, \nu=\nu^{\prime}$. Matrix $\Lambda^{*}$ of generators of $\iota^{\prime} d$ can be identified with the matrix $L^{-}$of F-R-T [19].

Owing to the unitarity of $U$, the equation $R U_{1} U_{2}=U_{2} U_{1} R$ is equivalent to

$$
U_{1}^{*} R^{-1} U_{2}=U_{2} R^{-1} U_{1}^{*}
$$

The Yang-Baxter equation (1.1) then implies 
Proposition 4.1. The $*$-Hopf algebra $\mathscr{A}_{q}(A N)$ is determined by the following relations:

$$
\begin{aligned}
R \Lambda_{1} \Lambda_{2} & =\Lambda_{2} \Lambda_{1} R, \\
\Lambda_{1}^{*} R^{-1} \Lambda_{2} & =\Lambda_{2} R^{-1} \Lambda_{1}^{*},
\end{aligned}
$$

and

$$
\begin{gathered}
\Delta \Lambda=\Lambda_{\otimes} \Lambda, \\
S(\Lambda)=\Lambda^{-1}, \\
\varepsilon(\Lambda)=I ;
\end{gathered}
$$

the matrix $\Lambda$ is upper triangle, the diagonal elements mutually commute and

$$
\prod \Lambda_{i i}=1
$$

The key role plays

\section{Proposition 4.2. It holds}

$$
\varrho\left(\Sigma_{\sigma} \Lambda_{\sigma k} \otimes U_{\jmath \sigma}\right) \varrho^{*}=\Sigma_{\sigma} \Lambda_{j \sigma} \otimes U_{\sigma k}
$$

for all $j, k$.

Proof. Rewrite (4.5) as

$$
\varrho\left(\Sigma_{\sigma} \Lambda_{\sigma k} \otimes U_{j \sigma}\right)=\left(\Sigma_{\sigma} \Lambda_{j \sigma} \otimes U_{\sigma k}\right) \varrho
$$

and embed this time $\mathscr{A}_{d} \otimes \mathscr{A}_{c}$ into $\operatorname{Lin}\left(\mathscr{C}_{c}\right)$. The canonical element $\varrho$ then corresponds to the identity and the elements $\varrho(u \otimes d)$ and $(u \otimes d) \varrho$ correspond to the mappings

$$
c \rightarrow((\mathrm{id} \otimes\langle u, \cdot\rangle) \Delta c) d
$$

and

$$
c \rightarrow d((\langle u, \cdot\rangle \otimes \mathrm{id}) \Delta c),
$$

respectively. Thus (4.5) can be rewritten once more:

$$
\Sigma_{\sigma}\left(\left(\mathrm{id} \otimes\left\langle\Lambda_{\sigma s}, \cdot\right\rangle\right) \Delta c\right) U_{j \sigma}=\Sigma_{\sigma} U_{\sigma s}\left(\left(\left\langle\Lambda_{\jmath \sigma}, \cdot\right\rangle \otimes \mathrm{id}\right) \Delta c\right)
$$

for all $c \in \ell_{q}(K)$ and all $j, s$.

Clearly, (4.6) is valid for $c=1$. The relations (3.1b), (4.3a) imply that it holds

$$
\begin{gathered}
\left(\mathrm{id} \otimes\left\langle\Lambda_{\sigma s}, \cdot\right\rangle\right) \Delta(c d)=\Sigma_{\nu}\left(\mathrm{id} \otimes\left\langle\Lambda_{\nu s}, \cdot\right\rangle\right) \Delta(c)\left(\mathrm{id} \otimes\left\langle\Lambda_{\sigma \nu}, \cdot\right\rangle\right) \Delta(d), \\
\left(\left\langle\Lambda_{j \nu}, \cdot\right\rangle \otimes \mathrm{id}\right) \Delta(c d)=\Sigma_{\sigma}\left(\left\langle\Lambda_{\sigma \nu}, \cdot\right\rangle \otimes \mathrm{id}\right) \Delta(c)\left(\left\langle\Lambda_{j \sigma}, \cdot\right\rangle \otimes \mathrm{id}\right) \Delta(d) .
\end{gathered}
$$

It follows easily that the relation (4.6) is satisfied for $c d$ provided it is satisfied both for $c$ and $d$. We conclude that it is enough to verify (4.6) only for the generators of the algebra $\mathscr{b}_{q}(K)$.

Letting $c=U_{k t}$ in (4.6) we get

$$
\Sigma_{\sigma, \nu}\left\langle\Lambda_{\sigma s}, U_{\nu t}\right\rangle U_{k \nu} U_{j \sigma}=\Sigma_{\sigma, \nu}\left\langle\Lambda_{j \sigma}, U_{k \nu}\right\rangle U_{\sigma s} U_{\nu t} .
$$

But owing to (4.1a) this is equivalent to

$$
U_{2} U_{1} R_{21}^{-1}=R_{21}^{-1} U_{1} U_{2} .
$$


Corollary 4.3. The matrix $T=U_{\otimes} \Lambda$ with entries belonging to the quantum double $\mathscr{D}(K)$ fulfills

$$
\begin{aligned}
R T_{1} T_{2} & =T_{2} T_{1} R, \\
T_{1}^{*} R^{-1} T_{2} & =T_{2} R^{-1} T_{1}^{*},
\end{aligned}
$$

and

$$
\begin{gathered}
\Delta T=T_{\otimes}^{\cdot} T, \\
S(T)=T^{-1}, \\
\varepsilon(T)=I .
\end{gathered}
$$

The same relations are also fulfilled by $T^{\prime}=\Lambda_{\otimes} U$ in $\mathscr{D}^{\prime}(K)$.

Remark. The identity (4.5) means that

$$
\Phi(T)=T^{\prime} .
$$

One can regard the $*$-Hopf algebras $\mathscr{A}_{q}(K)$ and $\mathscr{C}_{q}(A N)$ as being embeded into $\mathscr{D}$ and omit the sign of tensor product. Then each element from $\mathscr{A}_{q}(K)$ commutes with any element from $\mathscr{\ell}_{q}(A N)$. The entries of the matrix $T=U \Lambda$ generate some *-subalgebra $\mathscr{G}$ in $\mathscr{D}$ which is also sub-coalgebra owing to (4.8a). Let us now check the relation between $\mathscr{G}$ and $\mathscr{D}$.

To meet this goal we shall need a version of the non-commutative Gram-Schmidt orthogonalization process. Let us introduce the following property of completeness of a $*$-algebra,$f$ :

(*) If some positive element $u \in \mathscr{b}$ is represented by an invertible operator in all *-representations of $\mathscr{b}$ then $u$ is invertible in $\mathscr{t}$.

Lemma 4.4. Let to be a unital *-algebra having the property (*) and $F$ be a matrix from $\operatorname{Mat}(n) \otimes \mathscr{C}$ having a left inversion. Denote by $f_{1}, \ldots, f_{n}$ the columns of $F$. Then there exist orthogonal vectors $g_{1}, \ldots, g_{n}$ from $\mathscr{A}^{n}, g_{i}^{*} \cdot g_{j}=0$ for $i \neq j$, and matrices $S^{(k)} \in \operatorname{Mat}(n) \otimes \mathscr{A}, k=1, \ldots, n$, with units on the diagonal and with all nondiagonal elements equal to zero possibly except $S_{i j}^{(k)}, 1 \leq i<j \leq k$, such that it holds

$$
F^{(k)}:=F S^{(k)}=\left(g_{1}, \ldots, g_{k}, f_{k+1}, \ldots, f_{n}\right) .
$$

Proof. The property $(*)$ implies that if $F$ has a left inversion then the diagonal elements of $F^{*} F$ are invertible. Taking into account this observation and noting that matrices $S^{(k)}$ of the described type are manifestly invertible we can find the orthogonal vectors $g_{j}$ recursively:

$$
g_{1}=f_{1}, \quad g_{m+1}=f_{m+1}-\sum_{k=1}^{m} g_{k}\left(g_{k}^{*} \cdot g_{k}\right)^{-1}\left(g_{k}^{*} \cdot f_{m+1}\right) .
$$

This orthogonalization process can be applied to the matrix $T$ to decompose it into orthogonal and upper triangle parts. It follows that if the algebra $\mathscr{G}$ is completed by the square roots of the positive invertible elements lying on the diagonal of $\left(T^{(n)}\right)^{*} T^{(n)}$ we get the whole double $\mathscr{D}(K)$.

Let us now extend the Hopf algebra $\mathscr{C}_{q}^{\text {hol }}(G)$ to involve also quantum antiholomorphic functions on $G$. The resulting *-Hopf algebra $\mathscr{b}_{q}(G)$ is defined by the relations valid for $\mathscr{A}_{q}^{\mathrm{hol}}(G)$ and, in addition, by the relation $(4.7 \mathrm{~b})$. The dual $*$-Hopf algebra 
$U_{h}\left(\mathfrak{g}_{\mathbb{R}}\right)$ is a deformation of the enveloping algebra of the complexified Lie algebra $\mathfrak{g}_{\mathbb{R}} \otimes \mathbb{C}$. Its detailed description should be established but we shall not need it for our purposes. Nevertheless, it must be again valid that to the $i^{\text {th }}$ simple root there corresponds an embedding

$$
\check{\varphi}_{\imath, h}: U_{h}\left(\mathfrak{s l}(2)_{\mathbb{R}}\right) \rightarrow U_{h}\left(\mathfrak{g}_{\mathbb{R}}\right)
$$

and that the ranges $\operatorname{Ran}\left(\check{\varphi}_{i, h}\right)$ generate $U_{h}\left(\mathfrak{g}_{\mathbb{R}}\right)$.

Owing to Corollary 4.3 we have a morphism of $*$-Hopf algebras

$$
\kappa: /_{q}(G) \rightarrow \mathscr{L}(K) .
$$

Proposition 4.5. The homomorphism $\kappa$ is injective.

Proof. It is sufficient to show that the dual morphism $\breve{\kappa}$ is surjective. Note that to the $i^{\text {th }}$ simple root there corresponds also a morphism $\mathscr{C}(K) \rightarrow \mathscr{C}(S U(2))$. According to the results of [17], $(S U(2))$ can be identified with (more precisely, regarded as a completion of $). h_{q}(S L(2, \mathbb{C}))$. Thus we get a commutative diagram

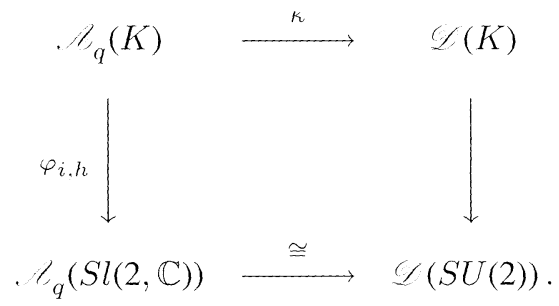

Considering now the dual diagram we see that $\operatorname{Ran}(\breve{\kappa})$ contains $\operatorname{Ran}\left(\breve{\varphi}_{\imath, h}\right)$ for all $i$ and hence $\operatorname{Ran}(\check{\kappa})=U_{h}\left(\mathfrak{g}_{\mathbb{R}}\right)$. Q.E.D.

According to this proposition, $\mathscr{Z}(K)$ can be regarded as a completion of . $/_{q}(G)$ and the relation $T=U \Lambda$ replaces the Iwasawa decomposition in the quantum case.

Remark. We note that the relation (4.7b) entering the definition of. ${ }_{q}(G)$ was discovered rather recently $[16,6]$. Its classical counterpart is given by $(2.12)$. What we wanted here to demonstrate is that it follows in a straightforward manner from the construction of the double group. Proposition 4.5 should be compared with results of [6] where the dual $*$-Hopf algebra to . ${ }_{1} q(G)$ [defined by relations $(4.7 \mathrm{a}, \mathrm{b})$ ] was constructed. This is in fact the quantum enveloping algebra of $\mathfrak{g}_{\mathbb{R}}$ denoted by $U_{h}\left(\mathfrak{g}_{\mathbb{R}}\right)$ above. The resulting algebra can be easily recognized as the dual $*$-Hopf algebra $\mathscr{L}(K)^{*}$ to the quantum double $\mathscr{L}(K)$. Following general ideas of F-R-T [8], $\mathscr{L}(K)^{*}$ is constructed again as a quantum double,

$$
\mathscr{Y}(K)^{*}=\cdot \mathscr{H}_{q}(K)^{*} \otimes \cdot \mathscr{l}_{q}(A N)^{*},
$$

now with trivial comultiplication and twisted multiplication. $\mathscr{L}(K)^{*} \otimes \mathscr{L}(K)$ possesses a universal element $\mathscr{T}$. With a knowledge of fundemental representation $\tau$ of $\mathscr{L}(K)^{*}$ such that

$$
(\tau \otimes \mathrm{id}) . T=T
$$

one could reduce the Iwasawa decomposition of $T$ to the factorization problem valid generally in any quantum double. 


\section{Quantum Leaf on $S U_{q}(2)$}

The first step is to introduce a quantum counterpart to the Poisson leaf $X$ (2.19), (2.20) on $S U_{q}(2)$. This quantum group is generated by four generators,

$$
U=\left(\begin{array}{cc}
a & b \\
-q^{-1} b^{*} & a^{*}
\end{array}\right), \quad q \in(0,1)
$$

satisfying relations

$$
\begin{gathered}
a b=q b a, \quad a b^{*}=q b^{*} a, \quad b b^{*}=b^{*} b, \\
a a^{*}-a^{*} a=\left(q^{-2}-1\right) b^{*} b, \quad a a^{*}+b b^{*}=1 .
\end{gathered}
$$

In the classical case the coordinate $z$ on $X$ is equal to $-U_{11} / U_{21}$. The quantum leaf should be described as a factor algebra $\mathscr{A}_{q}(S U(2)) / \mathscr{T}_{w}$ such that the image of the element $b$ is invertible (possibly after some completion). Set

$$
z=\left(b^{*}\right)^{-1} a .
$$

The elements $z$ and $z^{*}$ generate the $*$-algebra of quantum functions on the leaf and, as an easy computation shows, satisfy the relation

$$
1+z z^{*}=q^{-2}\left(1+z^{*} z\right) \text {. }
$$

More formally, denote by $\mathscr{L}$ the factor algebra of the algebra of formal power series in two non-commuting variables $z, z^{*}$ by the relation (5.4). The "restriction" morphism of $*$-algebras

$$
\psi_{w}: \mathscr{C}_{q}(S U(2)) \rightarrow \mathscr{L}
$$

is defined by

$$
\begin{gathered}
\psi_{w}(a)=\left(1+z z^{*}\right)^{-1 / 2} z \\
\psi_{w}(b)=\left(1+z z^{*}\right)^{-1 / 2} .
\end{gathered}
$$

The kernel

$$
\mathscr{T}_{w}:=\operatorname{Ker}\left(\psi_{w}\right)
$$

is a two-sided ideal in $\mathscr{C}_{q}(S U(2))$.

It is possible to introduce differential calculus on $\mathscr{b}$ determined by the rules

$$
\begin{gathered}
\partial_{z} z=1+q^{2} z \partial_{z}, \quad \partial_{z} z^{*}=q^{2} z^{*} \partial_{z}, \\
\partial_{\bar{z}} z=q^{-2} z \partial_{\bar{z}}, \quad \partial_{\bar{z}} z^{*}=1+q^{-2} z^{*} \partial_{\bar{z}},
\end{gathered}
$$

and consequently,

$$
\partial_{\bar{z}} \partial_{z}=q^{2} \partial_{z} \partial_{\bar{z}}
$$

Owing to (5.4), every element $f$ from $\mathscr{L}$ can be normally ordered,

$$
f=\sum \sum f_{j k}\left(z^{*}\right)^{j} z^{k}, \quad f_{j k} \in \mathbb{C} .
$$

This enables one to define a functional $\varepsilon_{w}$ on $\mathscr{L}$,

$$
\varepsilon_{w}(f)=f_{00} .
$$


Differential operators of finite order with constant coefficients form the dual vector space $\mathscr{X}$; the pairing between $\mathscr{Z}$ and $\mathscr{L}$ is defined by $(\xi \in \mathscr{B}, f \in \mathscr{L})$

$$
\langle\xi, f\rangle=\varepsilon_{w}(\xi \cdot f)
$$

particularly,

$$
\langle 1, f\rangle=\varepsilon_{w}(f)
$$

One can easily check that

$$
\left\langle\partial_{z}^{k} \partial_{\bar{z}}^{j},\left(z^{*}\right)^{\sigma} z^{\nu}\right\rangle=q^{\frac{1}{2}(k-j)(k+\jmath-1)}[j] ![k] ! \delta_{\jmath \sigma} \delta_{k \nu}
$$

$\left\{\partial_{z}^{k} \partial_{\bar{z}}^{j}\right\}_{\jmath, k}$ could be chosen for a basis in $\mathscr{C}$.

The pairing induces a coalgebra structure on $\mathscr{B}$ via

$$
\langle\Delta(\xi), f \otimes g\rangle=\langle\xi, f g\rangle \text {. }
$$

To describe the comultiplication explicitly we have to order normally the monomials $z^{k}\left(z^{*}\right)^{j}$. Put here and everywhere in what follows

$$
\lambda=q-q^{-1} \text {. }
$$

Lemma 5.1. It holds

$$
\begin{aligned}
z^{k}\left(z^{*}\right)^{j}= & q^{-2 j k} \sum_{s=0}^{\min (j, k)} q^{-\frac{1}{2} s(s+1)+(j+k) s} \frac{(-\lambda)^{s}}{[s] !} \frac{[j] ![k] !}{[j-s] ![k-s] !} \\
& \times\left(z^{*}\right)^{\jmath-s} z^{k-s} .
\end{aligned}
$$

Proposition 5.2. It holds

$$
\begin{aligned}
\Delta\left(\partial_{z}^{k} \partial_{\bar{z}}^{J}\right)= & \sum_{s=0}^{\infty} q^{-\frac{1}{2} s(s+1)} \frac{(-\lambda)^{s}}{[s] !} \sum_{\sigma=0}^{j} \sum_{\nu=0}^{k}\left[\begin{array}{l}
j \\
\sigma
\end{array}\right]\left[\begin{array}{l}
k \\
\nu
\end{array}\right] \\
& \times q^{\nu(k-\nu)-\sigma(j-\sigma)-2(j-\sigma+s) \nu} \partial_{z}^{\nu+s} \partial_{\bar{z}}^{\sigma} \otimes \partial_{z}^{k-\nu} \partial_{\bar{z}}^{j-\sigma+s},
\end{aligned}
$$

Particularly,

$$
\Delta(1)=\sum_{s=0}^{\infty} q^{-\frac{1}{2} s(s+1)} \frac{(-\lambda)^{s}}{[s] !} \partial_{z}^{s} \otimes \partial_{\bar{z}}^{s} .
$$

The next task is to compute the dual coalgebra morphism

$$
\check{\psi}_{w}: \mathscr{C} \rightarrow U_{h}(\mathfrak{s l}(2))
$$

Lemma 5.3. It holds

$$
\left\langle\partial_{z}^{\jmath} \partial_{\bar{z}}^{\jmath},\left(1-x z^{*} z\right)^{-1}\right\rangle=q^{-\jmath(\jmath-1)}([j] !)^{2} x^{j} / \prod_{i=1}^{j}\left(1-x\left(q^{-2 i}-1\right)\right),
$$

where $x$ is a complex parameter.

Proof. Start from

$$
\begin{aligned}
0 & =\left\langle\partial_{z}^{j} \partial_{\bar{z}}^{j},\left(1-x z^{*} z\right)\left(1-x z^{*} z\right)^{-1}\right\rangle \\
& =\left\langle\Delta\left(\partial_{z}^{j} \partial_{\bar{z}}^{j}\right),\left(1-x z^{*} z\right) \otimes\left(1-x z^{*} z\right)^{-1}\right\rangle,
\end{aligned}
$$

and then apply Proposition 5.2 and relation (5.10). Q.E.D. 
Lemma 5.4. It holds

$$
\left(z^{*} z\right)^{n}=\sum_{\jmath=1}^{n} q^{-\jmath(\jmath-1)} \sum_{\substack{k_{1} \geq 0 \\ k_{1}+\ldots+k_{j}=n-j}} \ldots \sum_{\substack{k_{\jmath} \geq 0 \\ n^{\prime}}}\left(q^{-2}-1\right)^{k_{1}} \ldots\left(q^{-2 j}-1\right)^{k_{j}}\left(z^{*}\right)^{\jmath} z^{\jmath} .
$$

Proof. Introduce complex functions

$$
F_{j}(x)=1 / \prod_{\imath=1}^{\jmath}\left(1-x\left(q^{-2 \imath}-1\right)\right) .
$$

Then by Lemma 5.3,

$$
\left\langle\partial_{z}^{j} \partial_{\bar{z}}^{j},\left(z^{*} z\right)^{k}\right\rangle=q^{-\jmath(j-1)}([j] !)^{2} \frac{1}{(k-j) !} \frac{d^{k-\jmath}}{d x^{k-\jmath}} F_{\jmath}(0) . \quad \text { Q.E.D. }
$$

Now we are able to order normally the elements $\psi_{w}(a), \psi_{w}(a)^{*}$ and $\psi_{w}(b)=$ $\psi_{w}(b)^{*}$ on the base of the following

Lemma 5.5. It holds

$$
\left(1+z^{*} z\right)^{-1 / 2}=1+\sum_{j=1}^{\infty}(-1)^{j} q^{\frac{3}{2} \jmath} \frac{\left[\frac{1}{2}\right]\left[\frac{3}{2}\right] \cdots\left[j-\frac{1}{2}\right]}{[j] !}\left(z^{*}\right)^{j} z^{j} .
$$

Proof. Using Lemma 5.4 and the identity

$$
(1+x)^{-1 / 2}=\sum_{n=0}^{\infty}(-1)^{n}\left(\begin{array}{c}
2 n \\
n
\end{array}\right)\left(\frac{x}{4}\right)^{n}
$$

we find that

$$
\left(1+z^{*} z\right)^{-1 / 2}=1+\sum_{\jmath=1}^{\infty} q^{-\jmath(\jmath-1)}\left(-\frac{1}{4}\right)^{j} C_{\jmath}\left(z^{*}\right)^{j} z^{j}
$$

where

$$
C_{\jmath}=\sum_{n=0}^{\infty}\left(-\frac{1}{4}\right)^{n}\left(\begin{array}{c}
2 n+2 j \\
n+j
\end{array}\right) \sum_{\substack{k_{1} \geq 0 \\
k_{1}+\ldots+k_{\jmath}=n}} \ldots \sum_{\substack{k_{j} \geq 0 \\
\text { 作 }}}\left(q^{-2}-1\right)^{k_{1}} \ldots\left(q^{-2 \jmath}-1\right)^{k_{\jmath}} .
$$

With the help of the interpolation formula

$$
x^{s}=\sum_{\imath=1}^{j} y_{\imath}^{s} \prod_{\substack{\nu=1 \\ \nu \neq i}}^{j} \frac{\left(x-y_{\nu}\right)}{\left(y_{i}-y_{\nu}\right)}, \quad \text { for } \quad s=0,1, \ldots, j-1,
$$

one is able to simplify (5.17) to get

$$
C_{j}=4^{\jmath} q^{\jmath(\jmath+1)} \prod_{\imath=1}^{\jmath}\left(1-q^{2 \imath-1}\right) / \prod_{i=1}^{\jmath}\left(1-q^{2 i}\right) .
$$


Corollary 5.6. It holds

$$
\begin{aligned}
\left\langle\check{\psi}_{w}\left(\partial_{z}^{k+1} \partial_{\bar{z}}^{k}\right), U\right\rangle & =(-1)^{k} q^{1+\frac{5}{2} k}[k+1] ! \prod_{i=0}^{k-1}\left[\frac{1}{2}+i\right]\left(\begin{array}{ll}
1 & 0 \\
0 & 0
\end{array}\right), \\
\left\langle\check{\psi}_{w}\left(\partial_{z}^{k} \partial_{\bar{z}}^{k+1}\right), U\right\rangle & =(-1)^{k} q^{1+\frac{1}{2} k}[k+1] ! \prod_{i=0}^{k-1}\left[\frac{1}{2}+i\right]\left(\begin{array}{ll}
0 & 0 \\
0 & 1
\end{array}\right), \\
\left\langle\check{\psi}_{w}\left(\partial_{z}^{k} \partial_{\bar{z}}^{k}\right), U\right\rangle & =(-1)^{k} q^{\frac{3}{2} k}[k] ! \prod_{i=0}^{k-1}\left[\frac{1}{2}+i\right]\left(\begin{array}{rr}
0 & q \\
-1 & 0
\end{array}\right),
\end{aligned}
$$

and all remaining values $\left\langle\check{\psi}_{w}\left(\partial_{z}^{k} \partial_{\bar{z}}^{\jmath}\right), U\right\rangle$ other than listed in (5.18) vanish.

Corollary 5.6 is still unsatisfactory for we want to identify the images $\breve{\psi}_{w}\left(\partial_{z}^{k} \partial_{\bar{z}}^{\jmath}\right)$ with some elements from the quantum enveloping algebra. Let us note that in the fundamental representation we are working with it holds

$$
\langle H, U\rangle=\left(\begin{array}{rr}
1 & 0 \\
0 & -1
\end{array}\right), \quad\left\langle X^{+}, U\right\rangle=\left(\begin{array}{ll}
0 & 1 \\
0 & 0
\end{array}\right), \quad\left\langle X^{-}, U\right\rangle=\left(\begin{array}{ll}
0 & 0 \\
1 & 0
\end{array}\right) .
$$

Set

$$
Y^{ \pm}=e^{ \pm h H / 2} X^{ \pm}
$$

Lemma 5.7. It holds

$$
\begin{gathered}
\check{\psi}_{w}\left(\partial_{z}^{k}\right)=(-1)^{k} q^{\frac{1}{2} k(2 k+1)}\left(Y^{+}\right)^{k} \check{\psi}_{w}(1), \\
\check{\psi}_{w}\left(\partial_{\check{z}}^{k}\right)=q^{\frac{1}{2} k}\left(Y^{-}\right)^{k} \check{\psi}_{w}(1) .
\end{gathered}
$$

Proof. We shall prove (5.20a), the second relation can be shown analogously. Equation (5.20a) means that

$$
\left\langle\check{\psi}_{w}\left(\partial_{z}^{k}\right), U_{1} \ldots U_{n}\right\rangle=(-1)^{k} q^{\frac{1}{2} k(2 k+1)}\left\langle Y^{+}, U_{1} \ldots U_{n}\right\rangle^{k}\left\langle\check{\psi}_{w}(1), U_{1} \ldots U_{n}\right\rangle,
$$

for all $n$ and arbitrary $k$. Clearly,

$$
\left\langle Y^{+}, U_{1} \ldots U_{n}\right\rangle=\sum_{i=1}^{n} I \otimes \ldots \otimes I \otimes Y_{(i)}^{+} \otimes e^{h H} \otimes \ldots \otimes e^{h H},
$$

where $Y^{+}$and $H$ on the RHS should be replaced by their representative in the fundamental representation.

We proceed by induction in $n$. The equality (5.21) with $n=1$ follows from Corollary 5.6. To perform the induction step $n \rightarrow n+1$ we take into account the fact that $\check{\psi}_{w}$ is a coalgebra morphism and apply the identity (5.15) and a particular case of (5.14), namely

$$
\Delta\left(\partial_{z}^{k}\right)=\sum_{s=0}^{\infty} q^{-\frac{1}{2} s(s+1)} \frac{(-\lambda)^{s}}{[s] !} \sum_{\nu=0}^{k}\left[\begin{array}{l}
k \\
\nu
\end{array}\right] q^{\nu(k-\nu)-2 s \nu} \partial_{z}^{\nu+s} \otimes \partial_{z}^{k-\nu} \partial_{\bar{z}}^{s} .
$$

Thus we are able to compute

$$
\left\langle\xi, U_{1} \ldots U_{n} U_{n+1}\right\rangle=\left\langle\Delta(\xi), U_{1} \ldots U_{n} \otimes U_{n+1}\right\rangle
$$


with $\xi=\breve{\psi}_{w}\left(\partial_{z}^{k}\right)$ and $\xi=\breve{\psi}_{w}(1)$ and verify consequently the identity (5.21) also with $n$ replaced by $n+1$. We omit remaining calculations which are straightforward though a bit tedious and only note that the following identity turns out to be helpful

$$
\sum_{s=0}^{k} q^{-k s+\frac{1}{2} s^{2}+s} \lambda^{s}\left[\begin{array}{l}
k \\
s
\end{array}\right] \prod_{i=0}^{s-1}\left[\frac{1}{2}+i\right]=q^{k} \text {. Q.E.D. }
$$

\section{Quantum Orbit Related to Quantum Weyl Element}

Now we are able to state a relation to the quantum Weyl element which was introduced in [11] and [13]. Below we follow notation of the paper [11]. Complete $U_{h}(\mathfrak{s l}(2))$ with an element $\check{W}$ unambiguously determined by the relations

$$
\langle\check{W}, U\rangle=\left(\begin{array}{rr}
0 & q \\
-1 & 0
\end{array}\right)
$$

and

$$
\Delta(\breve{W})=\tilde{R}_{21} \breve{W} \otimes \check{W}
$$

where

$$
\tilde{R}_{12}=\sum_{s=0}^{\infty} q^{\frac{1}{2} s(s+1)} \frac{\lambda^{s}}{[s] !}\left(Y^{-}\right)^{s} \otimes\left(Y^{+}\right)^{s} .
$$

The quantum Weyl element is defined by

$$
W=\exp \left(-\frac{h}{4} H^{2}\right) \check{W}
$$

and fulfills

$$
\Delta(W)=R_{21}^{u} W \otimes W
$$

where

$$
R_{12}^{u}=\exp \left(-\frac{h}{2} H \otimes H\right) \tilde{R}_{12}
$$

is the universal $R$-matrix.

Proposition 6.1.. It holds

$$
\check{\psi}_{w}(1)=\check{W} .
$$

Proof. It is enough to apply Lemma 5.7 to the identitiy (5.15) and the result compare with (6.2). Q.E.D.

The morphism $\breve{\psi}_{w}$ can be computed also in the general case.

Proposition 6.2. It holds

$$
\begin{aligned}
\check{\psi}_{w}\left(\partial_{z}^{k} \partial_{\bar{z}}^{j}\right)= & (-1)^{k} q^{\frac{1}{2} k(2 k+1)+\frac{1}{2} j} \sum_{s=0}^{\min (j, k)} q^{s(s-\jmath-k-1)} \frac{[j] !}{[j-s] !} \frac{[k] !}{[k-s] !} \\
& \times\left(Y^{+}\right)^{k-s} \prod_{r=1}^{s}\left(\frac{e^{h H}-q^{-2(s-r)}}{1-q^{-2 r}}\right)\left(Y^{-}\right)^{j-s} \check{W} .
\end{aligned}
$$


Proof. We start from the equation

$$
\Delta \breve{\psi}_{w}\left(\partial_{z}^{k}\right)=\breve{\psi}_{w}\left(\Delta \partial_{z}^{k}\right)
$$

The LHS can be computed with the help of the relation (5.20a) and Proposition 6.1.

$$
\begin{aligned}
\text { LHS }= & (-1)^{k} q^{\frac{1}{2} k(2 k+1)} \sum_{s=0}^{\infty} \sum_{\nu=0}^{k} q^{\frac{1}{2} s(s+1)+\nu(k-\nu)} \frac{\lambda^{s}}{[s] !}\left[\begin{array}{l}
k \\
\nu
\end{array}\right] \\
& \times\left(Y^{+}\right)^{\nu+s} \check{W} \otimes e^{\nu h H}\left(Y^{+}\right)^{k-\nu}\left(Y^{-}\right)^{s} \breve{W} .
\end{aligned}
$$

To get the RHS we apply (5.22) and again (5.20a, b)

$$
\mathrm{RHS}=\sum_{s=0}^{\infty} \sum_{\nu=0}^{k}(-1)^{\nu} q^{\frac{1}{2} s^{2}+\nu k+\frac{1}{2} \nu} \frac{\lambda^{s}}{[s] !}\left[\begin{array}{l}
k \\
\nu
\end{array}\right]\left(Y^{+}\right)^{\nu+s} \check{W} \otimes \check{\psi}_{w}\left(\partial_{z}^{k-\nu} \partial_{\bar{z}}^{s}\right) .
$$

For the elements $\left(Y^{+}\right)^{j}, j=0,1,2, \ldots$, are linearly independent in $U_{h}(\mathfrak{s l}(2))$ and $\breve{W}$ is invertible, one can compare the first multipliers in the tensor product to get

$$
\begin{aligned}
& (-1)^{\sigma} \sum_{s} q^{\frac{1}{2} s(s-1)+\frac{1}{2} \sigma} \frac{(-\lambda)^{s}}{[s] !}\left[\begin{array}{c}
k \\
s-\sigma
\end{array}\right] q^{(\sigma-s) k} \check{\psi}_{w}\left(\partial_{z}^{s-\sigma} \partial_{\check{z}}^{s}\right) \\
& =\sum_{s} q^{\frac{1}{2} s(s+1)-(s-\sigma)^{2}} \frac{\lambda^{s}}{[s] !}\left[\begin{array}{c}
k \\
s-\sigma
\end{array}\right] q^{(s-\sigma) k} e^{(k+\sigma-s) h H}\left(Y^{+}\right)^{s-\sigma}\left(Y^{-}\right)^{s} \check{W},
\end{aligned}
$$

where $k-\nu=s-\sigma$ and $\max (0, \sigma) \leq s \leq k+\sigma$. Summing both sides

$$
\sum_{k=0}^{t} q^{(t-1) k}(-1)^{t-k}\left[\begin{array}{l}
t \\
k
\end{array}\right] \times \operatorname{LHS}_{k}\left(\text { resp. } \mathrm{RHS}_{k}\right)
$$

and using the identity

$$
\sum_{k=0}^{t}(-1)^{t-k}\left[\begin{array}{l}
t \\
k
\end{array}\right]\left[\begin{array}{l}
k \\
j
\end{array}\right] q^{(t-\jmath-1) k}=q^{-t} \delta_{j t}
$$

$\left(\left[\begin{array}{l}n \\ m\end{array}\right]\right.$ is assumed to be equal to zero if $\left.m>n\right)$ and a quantum version of the binomial theorem we obtain the desired result. Q.E.D.

Corollary 6.3. The morphism $\check{\psi}_{w}$ is injective.

Denote by $\overline{U_{h}(\mathfrak{s l}(2))}$ the completion of the quantum enveloping algebra by the Weyl element. Then the range

$$
\mathscr{T}_{w}^{\vee}:=\operatorname{Ran}\left(\check{\psi}_{w}\right)
$$

is a sub-coalgebra in $\overline{U_{h}(\mathfrak{s l}(2))}$. Clearly,

$$
f \in \mathscr{T}_{w} \quad \text { iff } \quad\langle\xi, f\rangle=0 \text { for all } \xi \in \mathscr{T}_{w}^{\vee} .
$$

As the morphism $\check{\psi}_{w}$ is injective and $\mathscr{t}_{q}(S U(2)) / \mathscr{T}_{w}$ is naturally embedded into $\mathscr{L}$ the pairing between $\mathscr{C}$ and $\mathscr{A}_{q}(S U(2)) / \mathscr{T}_{w}$ is nondegenerate. In this sense $\mathscr{L}$ can be regarded as a completion of $\mathscr{C}_{q}(S U(2)) / \mathscr{T}_{w}$. 
It is possible to describe the left dressing transformation on $\mathscr{L}$ explicitly. Let

$$
\Lambda=\left(\begin{array}{cc}
\alpha & \beta \\
0 & \alpha^{-1}
\end{array}\right)
$$

be the fundamental representation of $\mathscr{C}_{q}(A N)$. The generators fulfill

$$
\begin{gathered}
\alpha \beta=q \beta \alpha, \quad \alpha \beta^{*}=q^{-1} \beta^{*} \alpha, \quad \alpha^{*}=\alpha, \\
{\left[\beta, \beta^{*}\right]=\left(1-q^{2}\right)\left(\alpha^{-2}-\alpha^{2}\right) .}
\end{gathered}
$$

The relation (4.5) means that

$$
\begin{gathered}
\varrho(\alpha \otimes a) \varrho^{*}=\alpha \otimes a-q^{-1} \beta \otimes b^{*}, \\
\varrho\left(\alpha \otimes b^{*}\right) \varrho^{*}=\alpha^{-1} \otimes b^{*}, \\
\varrho\left(\left(\beta \otimes a+\alpha^{-1} \otimes b\right) \varrho^{*}=\beta \otimes a^{*}+\alpha \otimes b,\right. \\
\varrho\left(-q^{-1} \beta \otimes b+\alpha^{-1} \otimes a^{*}\right) \varrho^{*}=\alpha^{-1} \otimes a^{*} .
\end{gathered}
$$

If

$$
L_{w}: \mathscr{B} \rightarrow \mathscr{C}_{q}(A N) \otimes \mathscr{B}
$$

is the factor action then from $(6.13 \mathrm{a}, \mathrm{b})$ and (5.3) it easily follows that

$$
L_{w}(z)=\alpha^{2} \otimes z-\beta \alpha \otimes 1
$$

One can also check by a direct computation that the elements $L_{w}(z), L_{w}(z)^{*}$ satisfy (5.4).

Next we want to discuss briefly in what sense the quantum leaf can be regarded as a quantum dressing orbit. To this end we introduce the dual dressing transformation

$$
L_{w}^{\vee}: U_{h}(\mathfrak{a n}) \otimes \mathscr{B} \rightarrow \mathscr{C} .
$$

The *-Hopf algebra $U_{h}(\mathfrak{a n})$ is dual to $\mathscr{A}_{q}(A N)$, coincides with $\mathscr{A}_{q}(S U(2))$ as a coalgebra and is opposite to $\mathscr{t}_{q}(S U(2))$ as a $*$-algebra. Redenote the generators of $\mathscr{C}_{q}(S U(2))$ as follows: $a$ as $F^{+}, a^{*}$ as $F^{-}, \lambda^{-1} q^{-3 / 2} b^{*}$ as $K^{+},-\lambda^{-1} q^{1 / 2} b$ as $K^{-}$. Then it holds

$$
\begin{aligned}
& \left\langle F^{ \pm}, \Lambda\right\rangle=\left\langle F^{ \pm}, \Lambda^{*}\right\rangle=\left(\begin{array}{cc}
q^{\mp 1 / 2} & 0 \\
0 & q^{ \pm 1 / 2}
\end{array}\right), \\
& \left\langle K^{+}, \Lambda\right\rangle=\left(\begin{array}{ll}
0 & 1 \\
0 & 0
\end{array}\right) \quad\left\langle K^{+}, \Lambda^{*}\right\rangle=0, \\
& \left\langle K^{-}, \Lambda\right\rangle=0, \quad\left\langle K^{-}, \Lambda^{*}\right\rangle=\left(\begin{array}{ll}
0 & 0 \\
1 & 0
\end{array}\right),
\end{aligned}
$$

further,

$$
\begin{gathered}
F^{+} K^{ \pm}=q K^{ \pm} F^{+}, \quad F^{-} K^{ \pm}=q^{-1} K^{ \pm} F^{-}, \quad K^{+} K^{-}=K^{-} K^{+} \\
F^{+} F^{-}-\lambda^{2} q K^{+} K^{-}=1=F^{-} F^{+}-\lambda^{2} q^{-1} K^{+} K^{-} \\
\left(K^{ \pm}\right)^{*}=-q^{\mp 2} K^{\mp}, \quad\left(F^{ \pm}\right)^{*}=F^{\mp}
\end{gathered}
$$

and finally,

$$
\Delta\left(K^{ \pm}\right)=K^{ \pm} \otimes F^{ \pm}+F^{\mp} \otimes K^{ \pm}, \quad \Delta\left(F^{ \pm}\right)=F^{ \pm} \otimes F^{ \pm}+\lambda^{2} K^{\mp} \otimes K^{ \pm} .
$$

Lemma 6.4. It holds

$$
L_{w}^{\vee}\left(\left(K^{+}\right)^{\nu}\left(K^{-}\right)^{\sigma} \otimes 1\right)=(-1)^{\nu+\sigma} q^{\nu \sigma+\frac{1}{2} \sigma^{2}-\frac{1}{2} \nu^{2}} \partial_{z}^{\nu} \partial_{\bar{z}}^{\sigma}
$$


Proof. Identifying $U_{h}(\mathfrak{a n})$ with $\mathscr{t}_{\mathrm{q}}(S U(2))$ as a coalgebra we have, in view of (6.14),

$$
\begin{aligned}
\left\langle L_{w}^{\vee}\left(U_{1} \ldots U_{n} \otimes 1\right),\left(z^{*}\right)^{j} z^{k}\right\rangle= & \left\langle U_{1} \ldots U_{n} \otimes 1, L_{w}\left(z^{*}\right)^{\jmath} L_{w}(z)^{k}\right\rangle \\
= & (-1)^{j+k}\left(\left\langle\alpha, U_{1} \ldots U_{n}\right\rangle\left\langle\beta^{*}, U_{1} \ldots U_{n}\right\rangle\right)^{j} \\
& \times\left(\left\langle\beta, U_{1} \ldots U_{n}\right\rangle\left\langle\alpha, U_{1} \ldots U_{n}\right\rangle\right)^{k} .
\end{aligned}
$$

Bearing in mind that the multiplication in $\mathscr{A}_{q}(S U(2))$ is now taken in the reversed order one easily finds that

$$
\begin{gathered}
\left\langle\alpha, U_{1} \ldots U_{n}\right\rangle=e^{h H / 2} \otimes \ldots \otimes e^{h H / 2} \\
\left\langle\beta, U_{1} \ldots U_{n}\right\rangle=q^{1 / 2} \sum_{i=1}^{n} e^{-h H / 2} \otimes \ldots \otimes e^{-h H / 2} \otimes X^{-} \otimes e^{h H / 2} \otimes \ldots \otimes e^{h H / 2}
\end{gathered}
$$

where $H$ and $X^{-}$on the RHS should be replaced by their two-dimensional representatives. As we are interested only in the values $L_{w}^{\vee}\left(\left(b^{*}\right)^{\nu} b^{\sigma} \otimes 1\right)$ it is enough to compare the coefficients standing on both sides at the matrices $X^{\omega_{1}} \otimes \ldots \otimes X^{\omega_{n}}$, where $\omega_{\imath}= \pm$. This task reduces to some combinatorics utilizing commutation relations. We again omit some tedious but straightforward computations and restrict ourselves only to recalling a helpful identity

$$
q^{-\frac{1}{2} n(n-1)} \sum_{\pi \in S_{n}} q^{22(\pi)}=[n] !,
$$

where $2(\pi)$ designates the number of transpositions in permuation $\pi$. Q.E.D.

Corollary 6.5. It holds

$$
L^{\vee}\left(U_{h}(\mathfrak{a n}) \otimes \check{W}\right)=\mathscr{T}_{w}^{\vee}
$$

Proof. By Lemma 6.4, $L_{w}^{\vee}\left(U_{h}(\mathfrak{a n}) \otimes 1\right)=\mathscr{B}^{*}$. The result then follows from Proposition 6.1 and the commutative diagram

$$
\begin{aligned}
& U_{h}(\mathfrak{a n}) \otimes \mathscr{X} \quad \stackrel{L_{w}^{\vee}}{\longrightarrow} \quad \mathscr{C} \\
& \operatorname{id} \otimes \check{\psi}_{w} \downarrow\left\lfloor\check{\psi}_{w} \quad\right. \text { Q.E.D. } \\
& U_{h}(\mathfrak{a n n}) \otimes U_{h}(\mathfrak{s l}(2)) \longrightarrow L^{\vee} \quad U_{h}(\mathfrak{s l}(2)) .
\end{aligned}
$$

In the classical case, the set on the LHS in (6.20) involves in fact all differential operators of finite order evaluated at the point $W=\left(\begin{array}{rr}0 & 1 \\ -1 & 0\end{array}\right) \in S U(2)$ and tangent to the dressing orbit. As the orbit is real analytic and connected it is determined by this set of differential operators unambiguously. On the other hand, the coalgebra $\mathscr{T}_{w}^{\mathrm{V}}$ determines the two-sided ideal $\mathscr{T}_{w} \subset \mathscr{A}_{q}(S U(2))$ according to (6.10) and $\mathscr{T}_{w}$ is the quantum counterpart to the Poisson leaf. Thus the relation (6.20) suggests a quantum analogy to the most distinguishing property of the classical dressing transformation according to which the dressing orbits coincide with the Poisson leaves.

We conclude this section with a short description of quantum leaves on a compact group $K_{q}$ in the general case. Let us complete $U_{h}(\mathfrak{g})$ by the quantum Weyl elements 
$W_{i}$ and $\check{W}_{i}$ corresponding to the simple roots and, moreover, by the elements of the maximal torus $M \subset K$ in accordance with the rules

$$
\begin{gathered}
\langle t, U\rangle=t, \\
\Delta t=t \otimes t .
\end{gathered}
$$

To each $t \in M$ there corresponds a one-dimensional *-representation $\tau$ of $\mathscr{t}_{q}(K)$, $\tau(f)=\langle t, f\rangle$. Denote by

$$
\Delta_{n}: \mathscr{A}_{q}(K) \rightarrow \mathscr{A}_{q}(K) \otimes \ldots \otimes \mathscr{A}_{q}(K) \quad(n \text { copies })
$$

a $*$-algebra morphism defined recursively,

$$
\Delta_{1}=\mathrm{id}, \quad \Delta_{n+1}=(\mathrm{id} \otimes \ldots \otimes \mathrm{id} \otimes \Delta) \Delta_{n} .
$$

Then to each Weyl element with a reduced decomposition $W=W_{i_{1}} \ldots W_{\imath_{k}}$ and to each element $t \in M$ there corresponds a quantum leaf

$$
\mathscr{L}_{w t}=\mathscr{L}_{i_{1}} \otimes \ldots \otimes \mathscr{L}_{i_{k}}
$$

with the "restriction" homomorphism

$$
\psi_{w t}:=\left(\psi_{i_{1}} \otimes \ldots \otimes \psi_{i_{k}} \otimes \tau\right) \Delta_{k+1}: \mathscr{f}_{q}(K) \rightarrow \mathscr{D}_{w t},
$$

where

$$
\psi_{i}=\psi_{w_{i}} \circ \varphi_{i, h}: \mathscr{b}_{q}(K) \rightarrow \mathscr{L}_{i} .
$$

By the results of $[11,13]$, the image

$$
\check{\psi}_{w t}(1)=\check{W}_{i_{1}} \ldots \check{W}_{i_{k}} t \in \overline{U_{h}(\mathfrak{g})}
$$

does not depend on the choice of reduced decomposition. The above papers also provide communication relations between the quantum Weyl elements and the generators of $U_{h}(\mathfrak{g})$. This means, owing to Proposition 6.2, that we know the dual coalgebra morphism $\check{\psi}_{w t}$ explicitly.

\section{Irreducible $*$-Representations for Algebras of Quantum Functions on Compact Groups}

The case of $S U_{q}(2)$ was originally investigated in the papers by Woronowicz [31] and Vaksman and Soibel'man [28]. Particularly, it was found in [28] that every irreducible *-representation of $S U_{q}(2)$ is unitarily equivalent to one of the following two types [recall the relations $(5.1),(5.2)]$ :

(i) one-dimensional representations $\xi_{\varphi}$

$$
\xi_{\varphi}(a)=e^{-i \varphi}, \quad \xi_{\varphi}(b)=0, \quad \varphi \in \mathbb{R} / 2 \pi \mathbb{Z} .
$$

(ii) infinite-dimensional representations $\pi_{\varphi}$, given in an orthonormal basis

$$
\begin{gathered}
|n\rangle=A_{n}^{-1}\left(a^{*}\right)^{n}|0\rangle, \\
A_{n}^{2}=\left(1-q^{2}\right) \ldots\left(1-q^{2 n}\right), \quad n=0,1, \ldots,
\end{gathered}
$$

by formulas

$$
\begin{gathered}
\pi_{\varphi}(a)|0\rangle=0, \quad \pi_{\varphi}(a)|n\rangle=\left(1-q^{2 n}\right)^{1 / 2}|n-1\rangle, \quad n=1, \ldots, \\
\pi_{\varphi}(b)|n\rangle=-q^{n+1} e^{-i \varphi}|n\rangle .
\end{gathered}
$$


Representations unitarily equivalent to those of (ii) also appeared in [9] in connection with the Liouville model on lattice. Irreducible *-representations of $S U_{q}(3)$ were investigated in [5] and generalization to the $S U_{q}(n)$ case by the Verma module construction were given in [26]. A series of papers due to Soibel'man [22,23] and Vaksman and Soibel'man [27] led to the following general results:

(i) Let $\pi$ designate the representation given by (7.3) with $e^{i \varphi}=-1$ and set $\pi_{\imath}=\pi \circ \varphi_{\imath, h}$. Every irreducible $*$-representation of a simple compact quantum group is equivalent to

$$
\pi_{W \tau}=\left(\pi_{i_{1}} \otimes \ldots \otimes \pi_{i_{k}} \otimes \tau\right) \Delta_{k+1}
$$

where $\tau$ is some one-dimensional representation (all one-dimensional representations are of the form $\tau: U \rightarrow t$ with $t$ being an element of the maximal torus) and $W=W_{\imath_{1}} \otimes \ldots \otimes W_{\imath_{k}}$ is a reduced decomposition of some Weyl element. Different reduced decompositions of the same Weyl element lead to equivalent representations.

(ii) As it follows from the previous item and the description of symplectic leaves of the corresponding Poisson Lie structure there is one-to-one correspondence between symplectic leaves and irreducible $*$-representations.

As more times stressed by Vaksman and Soibel'man this situation is very similar to the famous method of orbits (geometric quantization) due to Kirillov, Kostant, and Souriau. Below we support this point of view by a impressively simple construction utilizing differential geometry of the quantum leaf. Besides, in the next section we present another construction, though leading to the same result, based on the so-called Berezin's quantization applied to the classical Poisson leaf.

We again start our description from the quantum leaf $\mathscr{L}$ on $S U_{q}(2)$ which was introduced in Sect. 5. To a complex variable $\eta$ relate a quantum differential operator $X_{\eta}$ of infinite order,

$$
X_{\eta}=\sum_{\jmath=0}^{\infty} q^{\frac{1}{2} \jmath(j-1)} \frac{\eta^{j}}{[j] !} \partial_{\bar{z}}^{j},
$$

and to a quantum function $f \in \mathscr{L}$ relate a formal power series

$$
F_{f}(\eta)=\left\langle X_{\eta}, f\right\rangle \in \mathbb{C}[[\eta]] .
$$

Provided this power series $F_{f}(\eta)$ is convergent it can be regarded as a holomorphic function on the classical Poisson leaf $X \cong \mathbb{C}$. In this way we get a vector space of holomorphic functions on $X$. At the same time, it is a carrier space of a representation $\pi$ of the algebra $\mathscr{A}_{q}(S U(2))$,

$$
\pi(c) F_{f}=F_{\psi_{w}(c) f}
$$

The representation $\pi$ is well defined. Actually, the equality $F_{f}=F_{f^{\prime}}$ holds if and only if

$$
\left\langle\partial_{\bar{z}}^{\jmath}, f\right\rangle=\left\langle\partial_{\bar{z}}^{\jmath}, f^{\prime}\right\rangle \text { for all } j .
$$

But whenever this case happens then, owing to (5.14),

$$
\begin{aligned}
\left\langle\partial_{\bar{z}}^{\jmath}, \psi_{w}(c) f\right\rangle & =\left\langle\Delta\left(\partial_{\bar{z}}^{\jmath}\right), \psi_{w}(c) \otimes f\right\rangle \\
& =\sum_{s=0}^{\infty} q^{-\frac{1}{2} s(s+1)} \frac{(-\lambda)^{s}}{[s] !} \sum_{\sigma=0}^{\jmath}\left[\begin{array}{l}
j \\
\sigma
\end{array}\right] q^{-\sigma(\jmath-\sigma)}\left\langle\partial_{z}^{s} \partial_{\bar{z}}^{\sigma}, \psi_{w}(c)\right\rangle\left\langle\partial_{\bar{z}}^{\jmath-\sigma+s}, f\right\rangle \\
& =\left\langle\partial_{\bar{z}}^{\jmath}, \psi_{w}(c) f^{\prime}\right\rangle .
\end{aligned}
$$


Further, we introduce a scalar product,

$$
\left\langle F_{f}(\eta) \mid F_{g}(\eta)\right\rangle=\left\langle 1, f^{*} g\right\rangle .
$$

Because of (5.15) and the relation

$$
\left\langle\partial_{z}^{s}, f^{*}\right\rangle=q^{s(s-1)}\left\langle\partial_{\bar{z}}^{s}, f\right\rangle^{*},
$$

we have

$$
\begin{aligned}
\left\langle F_{f}(\eta) \mid F_{g}(\eta)\right\rangle & =\left\langle\Delta(1), f^{*} \otimes g\right\rangle \\
& =\sum_{s=0}^{\infty} q^{\frac{1}{2} s(s-3)} \frac{(-\lambda)^{s}}{[s] !}\left\langle\partial_{\bar{z}}^{s}, f\right\rangle^{*}\left\langle\partial_{\bar{z}}^{s}, g\right\rangle .
\end{aligned}
$$

The RHS of (7.9) shows again that the result does not depend on the choice of $f$ and $g$. Let us recall that $q \in(0,1)$ and so $\lambda=q-q^{-1}<0$. It follows that if the norm $\left\|F_{f}\right\|$ for a nonzero element $f$ is finite then it is positive. Restricting the representation $\pi$ to the Hilbert space consisting of holomorphic functions with finite norms we get a $*$-representation:

$$
\begin{aligned}
\left\langle F_{f}(\eta) \mid \pi(c) F_{g}(\eta)\right\rangle & =\left\langle 1, f^{*} \psi_{w}(c) g\right\rangle=\left\langle 1,\left(\psi_{w}\left(c^{*}\right) f\right)^{*} g\right\rangle \\
& =\left\langle\pi\left(c^{*}\right) F_{f}(\eta) \mid F_{g}(\eta)\right\rangle .
\end{aligned}
$$

Set now

$$
f_{n}=q^{\frac{1}{2} n(n+1)} A_{n}^{-1}\left(z^{*}\right)^{n} \in Y
$$

and

$$
F_{n}(\eta)=\left\langle X_{\eta}, f_{n}\right\rangle=q^{\frac{1}{2} n(n+1)} A_{n}^{-1} \eta^{n}, \quad n=0,1,2, \ldots
$$

Then using Lemma 5.1 and the commutation relation

$$
\left(1+z z^{*}\right)^{-1 / 2} z^{*}=q z^{*}\left(1+z z^{*}\right)^{-1 / 2}
$$

one can compute

$$
\begin{aligned}
\pi\left(a^{*}\right) F_{n}(\eta) & =q^{\frac{1}{2} n(n+1)} A_{n}^{-1}\left\langle X_{\eta}, z^{*}\left(1+z z^{*}\right)^{-1 / 2}\left(z^{*}\right)^{n}\right\rangle \\
& =q^{\frac{1}{2}(n+1)(n+2)} A_{n}^{-1}\left\langle X_{\eta},\left(z^{*}\right)^{n+1}\right\rangle=\left(1-q^{2 n+2}\right)^{1 / 2} F_{n+1}(\eta), \\
\pi(a) F_{n}(\eta) & =q^{\frac{1}{2} n(n+1)} A_{n}^{-1}\left\langle X_{\eta},\left(1+z z^{*}\right)^{-1 / 2} z\left(z^{*}\right)^{n}\right\rangle \\
& =-\lambda[n] q^{\frac{1}{2}(n+1)} A_{n}^{-1}\left\langle X_{\eta},\left(z^{*}\right)^{n-1}\right\rangle=\left(1-q^{2 n}\right)^{1 / 2} F_{n-1}(\eta), \\
\pi(b) F_{n}(\eta) & =q^{\frac{1}{2} n(n+1)} A_{n}^{-1}\left\langle X_{\eta},\left(1+z z^{*}\right)^{-1 / 2}\left(z^{*}\right)^{n}\right\rangle \\
& =q^{\frac{1}{2}(n+1)(n+2)} A_{n}^{-1}\left\langle X_{\eta},\left(z^{*}\right)^{n}\right\rangle=q^{n+1} F_{n}(\eta) .
\end{aligned}
$$

Furthermore, we have

$$
\left\langle\eta^{j} \mid \eta^{k}\right\rangle=\left\langle 1, z^{\jmath}\left(z^{*}\right)^{k}\right\rangle=\delta_{\jmath k} q^{-\frac{1}{2} j(\jmath+1)}(-\lambda)^{j}[j] !=\delta_{\jmath k} q^{-j(j+1)} A_{\jmath}^{2},
$$

and so $\left(F_{n}(\eta)\right)_{n}$ is an orthonormal basis. Identifying $F_{n}$ with the vectors $|n\rangle$ in (7.2) we get exactly the representation (7.3) with $e^{\imath \varphi}=-1$. 
Having established the representation we can rewrite all the formulae only in terms of holomorphic functions, i.e., without any reference to the quantum leaf. First, introduce an operator $L_{\kappa}$ depending on a positive parameter $\kappa$ by

$$
L_{\kappa} F(\eta)=F(\kappa \eta) \text {. }
$$

Then it is easy to verify that

$$
\begin{gathered}
\pi(a)=\frac{1}{\eta}\left(L_{q^{-1}}-L_{q}\right), \\
\pi\left(a^{*}\right)=q \eta L_{q}, \\
\pi(b)=\pi\left(b^{*}\right)=q L_{q} .
\end{gathered}
$$

To get a formula for the scalar product we express the reproducing kernel $K(u, \bar{v})$ corresponding to the basis $\left(F_{n}\right)$ with the help of Euler identity $(q \in(0,1))$,

$$
\begin{aligned}
K(u, \bar{v}) & :=\sum_{n=1}^{\infty} F_{n}(u) F_{n}(v)^{*}=1+\sum_{n=1}^{\infty} q^{n(n+1)} \frac{(u \bar{v})^{n}}{\left(1-q^{2}\right) \ldots\left(1-q^{2 n}\right)} \\
& =\prod_{k=1}^{\infty}\left(1+q^{2 k} u \bar{v}\right) .
\end{aligned}
$$

On the other hand, an application of the Jackson's $q$-integral yields the identity

$$
\sum_{n=-\infty}^{\infty} q^{2(j+1) n} \prod_{k=n}^{\infty}\left(1+q^{2 k}\right)^{-1}=q^{-\jmath(\jmath+1)}\left(1-q^{2}\right) \ldots\left(1-q^{2 j}\right) .
$$

In view of (7.9), we can write

$$
\langle F(\eta) \mid G(\eta)\rangle=\frac{1}{2 \pi} \int_{0}^{2 \pi} d \phi \int_{0}^{\infty} d \mu(r) K(\eta, \bar{\eta})^{-1} F(\eta)^{*} G(\eta),
$$

where $\eta=r e^{i \phi}$ and $d \mu(r)$ is a discrete measure on $\mathbb{R}^{+}$,

$$
d \mu(r)=\frac{r^{2}}{1+r^{2}} \sum_{n=-\infty}^{\infty} \delta\left(r-q^{n}\right) d r .
$$

The construction can be extended to a general quantum leaf $\mathscr{L}_{w t}$ in a straightforward manner. Now we set

$$
X_{\eta}=X_{\eta_{1}} \otimes \ldots \otimes X_{\eta_{k}}
$$

where $\eta=\left(\eta_{1}, \ldots, \eta_{k}\right)$ are coordinates on the classical Poisson leaf $X_{w} \cong \mathbb{C}^{k}$. If $f \in \mathscr{L}_{w t}, c \in \mathscr{b}_{q}(K), \psi_{w}$ is replaced by $\psi_{w t}$ and $\pi$ by $\pi_{w t}$ then the relations (7.6), (7.7), and (7.8) remain valid. Recalling the definition of the morphism $\psi_{w t}$ we have

$$
\begin{aligned}
\pi_{w t}(c) F_{f}(\eta) & =\left\langle X_{\eta_{1}} \otimes \ldots \otimes X_{\eta_{k}},\left(\psi_{\imath_{1}} \otimes \ldots \otimes \psi_{i_{k}} \otimes \tau\right) \Delta_{k+1}(c) f\right\rangle \\
& =\left(\pi_{i_{1}} \otimes \ldots \otimes \pi_{\imath_{k}} \otimes \tau\right) \Delta_{k+1}(c) F_{f}(\eta) .
\end{aligned}
$$

This means that $\pi_{w t}$ decomposes into a direct product in accordance with (7.4). 


\section{Quantization of Symplectic Leaves of the Poisson Lie Structure}

Here again we can confine ourselves to the simplest case, i.e., to the leaf on the group $S U(2)$ which is described by a pair of complex variables $(z, \bar{z})$ and the Poisson bracket (2.20). Traditionally, when a classical manifold is quantized (for a moment we denote by $f^{\wedge}$ an operator resulting from the quantization of a classical function $f$ ) it is assumed that a relation between Poisson bracket and commutator of the form

$$
\left[f^{\wedge}, g^{\wedge}\right]=\kappa\{f, g\}^{\wedge}
$$

is valid for functions $f, g$ from some prefered set of functions on the classical phase space. Here $\kappa$ is some nonzero real parameter playing the role of the Planck constant. The classical limit is obtained as $\kappa \rightarrow 0$. In our case we assume as preferred functions the coordinate variables $z, \bar{z}$ and as a quantization rule we take the normal ordering. It means that for an arbitrary monomial of the form $\bar{z}^{m} z^{n}$ we write

$$
\left(\bar{z}^{m} z^{n}\right)^{\wedge}=\left(\zeta^{*}\right)^{m} \zeta^{n},
$$

where we have used notation $z^{\wedge} \equiv \zeta, \bar{z}^{\wedge} \equiv \zeta^{*}$. Having this rules in mind we get a commutation relation for $\zeta$ and $\zeta^{*}$ in the form

$$
\left[\zeta, \zeta^{*}\right]=\kappa\left(1+\zeta^{*} \zeta\right) .
$$

It is easy to recognize in (8.3) the commutation relation (5.4) for $\zeta=\left(b^{*}\right)^{-1} a$ in which $\kappa=q^{-2}-1$. In analogy with the case of usual bosonic creation and annihilation operators one can realize the representation space $H$ for the irreducible *-representation $\pi$ (7.3) $\left(e^{\imath \phi}=-1\right)$ as a Hilbert space of analytic functions on the complex plane. Expressing

$$
\pi(a)=\left(1+\zeta \zeta^{*}\right)^{-1 / 2} \zeta, \quad \pi(b)=\left(1+\zeta \zeta^{*}\right)^{-1 / 2}
$$

we arrive again at the relations (7.14), (7.17). Such a realization is an analogue of the Bargmann representation of the Fock space for usual bosonic operators [2]. Let us stress also an analogy with Berezin's quantization on Kähler manifolds [3,15].

To proceed further in this analogy we recall the reproducing kernel defined by (7.15). For $F \in H$ we can write

$$
F(z)=\langle K(\bar{z}, \cdot) \mid F\rangle,
$$

where the variable $z$ in $K(\bar{z}, v)$ is assumed to be fixed. It can be easily seen that the function $\Phi_{\bar{v}}(z)=K(z, \bar{v})$ belongs to $H$ and from (7.17) it follows that a set of these functions with $v$ running over the whole complex plane constitutes an overcomplete system of functions on $H$ in the sense of Berezin [3]. In accordance with basic requirements (continuity and completeness) of [12] they can be called coherent states. It can be easily verified that these states are (unnormalized) eigenstates of the operator $\zeta$,

$$
\zeta \Phi_{\bar{v}}(z)=\bar{v} \Phi_{\bar{v}}(z) .
$$

In Dirac's notation $\Phi_{v}(z)=|v\rangle$,

$$
\begin{gathered}
|v\rangle=\sum_{n} q^{\frac{1}{2} n(n+1)} \frac{v^{n}}{A_{n}}|n\rangle, \\
F(z)=\langle\bar{z} \mid F\rangle,
\end{gathered}
$$


and the definition of inner product can be rewritten as a resolution of unity (understood in a week sense)

$$
I=\frac{1}{2 \pi} \int_{0}^{2 \pi} d \phi \int_{0}^{\infty} d \mu(r) K(z, \bar{z})^{-1}|\bar{z}\rangle\langle\bar{z}| .
$$

For later convenience we introduce a shorthanded notation for the inner product $\langle G \mid F\rangle=I_{z}(\widehat{G(z)} F(z))$. If we define according to Berezin [4] the covariant symbol of a linear operator $A$ on $H$ as

$$
A(z, \bar{z})=\langle\bar{z}|A| \bar{z}\rangle /\langle\bar{z} \mid \bar{z}\rangle
$$

we get a one-to-one correspondence between the operators and their covariant symbols [3],

$$
(A F)(z)=I_{v}(A(z, \bar{v}) F(v) K(z, \bar{v})),
$$

where $A(z, \bar{z})$ is defined by the analytic continuation of the covariant symbol. This correspondence generalizes the normal ordering rule given by (8.2). If now $A=A_{1} \cdot A_{2}$ we get for the covariant symbol of an operator $A$ the following expression:

$$
A(z, \bar{z})=I_{v}\left(A_{1}(z, \bar{v}) A_{2}(v, \bar{z}) K(z, \bar{v}) K(v, \bar{z}) K(z, \bar{z})^{-1}\right) .
$$

This formula can be used as a definition of the star product of two covariant symbols (functions on classical phase space) and can be rewritten in the following interesting form:

$$
\begin{aligned}
A(z, \bar{z}) & =\left(A_{1} * A_{2}\right)(z, \bar{z}) \\
& =\left.A_{1}\left(z+(1+z \bar{v}) \bar{v}^{-1}\left(-1+L_{q^{-2}}^{\bar{v}}\right), \bar{z}\right) A_{2}(z, \bar{v})\right|_{v=z},
\end{aligned}
$$

where the superscript in $L_{q^{-2}}^{\bar{v}}$ refers to the variable in which the operator acts. Expanding the right-hand side of the above equality in a Taylor series in $h$ we see that the correspondence principle in the form

$$
\{\cdot, \cdot\}=\lim _{h \rightarrow 0} 1 /(h)[\cdot, \cdot]
$$

holds. Let us remember also the formula for the trace of a bounded linear operator

$$
\operatorname{Tr} A=I_{z}(A(z, \bar{z})) \text {. }
$$

Coherent states as introduced here can be interpreted in terms of quantum differential operators in the following way. Let us consider a quantum differential operator $X_{\eta \bar{\varrho}}$ of infinite order

$$
X_{\eta \bar{\varrho}}=\sum_{j=0}^{\infty} q^{-\frac{1}{2}(k-\jmath)(k+j+1)} \frac{\varrho^{k}}{[k] !} \frac{\eta^{j}}{[j] !} \partial_{z}^{k} \partial_{\bar{z}}^{j}, \quad \eta, \varrho \in \mathbb{C} .
$$

Particularly, $X_{\eta}$ given by (7.5) is simply $X_{\eta 0}$. Considering $f \in \mathscr{L}$ as an operator we have owing to (5.10) and (8.6),

$$
\left\langle X_{\eta \bar{\varrho}}, f\right\rangle=\frac{\langle\bar{\eta}|f| \bar{\varrho}\rangle}{\langle\bar{\eta} \mid \bar{\varrho}\rangle}=f(\eta, \bar{\varrho}) .
$$

If $g \in A N$ and $g \cdot \eta$ denotes the classical dressing action we can introduce thanks to the correspondence between symbols and operators by formula

$$
\left(\tau_{g} f\right)(\eta, \bar{\eta})=\left\langle X_{\eta \bar{\eta}}, \tau_{g} f\right\rangle=\left\langle X_{g \eta, g \bar{\eta}}, f\right\rangle=f(g \eta, g \bar{\eta})
$$


a mapping $\tau_{g}: \mathscr{S} \rightarrow \mathscr{B}$. Let us note that this mapping is not an algebra homomorphism. The reason is because the classical dressing action is not Hamiltonian.

Attempting a unified approach Berezin defined quantization of a symplectic manifold $(M, \omega)$ ( $\omega$ is the symplectic form on the manifold $M$ ) as an associative algebra to with involution such that

(i) There is a family $\left\{\mathscr{C}_{h}\right\}$ of associative algebras with involution such that $h \in \mathbb{R}_{+}$, and the algebra $A$ consists of the functions

$$
f: \mathbb{R}^{+} \rightarrow \mathscr{A}: h \rightarrow f(h)
$$

taking values in $\mathscr{C}_{h}\left(\mathscr{A}=\underset{h \in R^{+}}{\oplus} \mathscr{A}_{h}\right)$. The multiplication and involution in $\mathscr{A}_{\text {, }}$ are connected with the multiplication and involution in $\mathscr{A}_{h}$ in the usual way

$$
(f * g)(h)=f(h) * g(h), \quad\left(f^{*}\right)(h)=(f(h))^{*} .
$$

The multiplication and involution in algebras . to and $A_{h}$ are denoted by the same symbols.

(ii) Correspondence principle. There exist an associative $*$-algebra homomorphism $\sigma$ of $A_{0}$ into the algebra $C(M, \mathbb{C})$ of $C$ functions on $M$ with standard operations of addition and pointwise multiplication such that for $g: h \rightarrow g(h)$,

$$
g(h)=h^{-1}\left(f_{1} * f_{2}-f_{2} * f_{1}\right)(h), \quad \sigma(g)=\left\{\sigma\left(f_{1}\right), \sigma\left(f_{2}\right)\right\}
$$

holds,

$$
\sigma\left(f^{*}\right)=\overline{\sigma(f)}
$$

where the bar stands for complex conjugation and for any two points $x_{1}$ and $x_{2}$ in $M$ there is a function $f(x)$ from $\sigma(\mathscr{A})$ such that $f\left(x_{1}\right) \neq f\left(x_{2}\right)$ [i.e., " $f$ separates points $x_{1}, x_{2}$ "; there are enough classical observables in $\sigma(\mathscr{l})$ to distinguish states].

As a special quantization of $(M, \omega)$ Berezin introduced a quantization with additional properties

(iii) (1) $\mathscr{A}_{h}$ consists of $C$ functions $f(x), x \in M$.

(2) $A_{b}$ consists of the functions $f(h, x)$, where $f(h, x) \in \mathscr{A}_{h}$ for fixed $h$.

(3) Homomorphism $\sigma: \mathscr{b} \rightarrow C(M, \mathbb{C})$ is given by the classical limit

$$
\sigma(f)=\lim _{h \rightarrow 0} f(h, x) .
$$

As noticed by Berezin all known special quantizations have also following two properties:

(iv) The algebra $\mathscr{l}$ contains a unity, which is a function $f(h, x) \equiv 1$.

(v) The algebra $\mathscr{t}_{h}$ possesses a trace, which is given by

$$
\operatorname{Tr} f=c \int f(x) d \mu(x),
$$

with $d \mu(x)$ being some measure on $M$ and $c$ some number factor.

Now from these definitions it is obvious that the correspondence between the operators and their covariant symbols as described above fulfills all requirements (i)-(v) for the special quantization of symplectic leaf $X$ of the Poisson Lie structure on $S U(2)$. The situation is very similar to Berezin's quantization for Kähler manifolds $[3,15]$ where coherent states play also a prominent role as well as to the geometric quantization on Kähler manifolds described in $[29,25]$ where the representation space is formed by polarized functions on classical phase space. In our case the dressing transformation replaces the group of motions [3] from the former case. 


\section{References}

1. Babelon, O., Bernard, D.: Dressing transformations and the origin of the quantum group symmetries. Preprint SPhT-91-016 (1991)

2. Bargmann, V.: Ann. Math. 48, 568 (1947)

3. Berezin, F.A.: General concept of quantization. Commun. Math. Phys. 40, 153 (1975)

4. Berezin, F.A.: Covariant and contravariant symbol of operators. Izv. Akad. Nauk USSR 36, 1134 (1972) (in Russian)

5. Bragiel, K.: The twisted $S U(3)$ group. Irreducible $*$-representations of the $C^{*}$-algebra $C\left(S_{\mu} U(3)\right)$ algebra. Lett. Math. Phys. 17, 37 (1989)

6. Drabant, B., Schlieker, M., Weich, W., Zumino, B.: Complex quantum groups and their enveloping algebras. Preprint MPI-PTh/91-75 (1991)

7. Drinfel'd, V.G.: Quantum groups. In: Proc. at the Intl. Cong. of Math. 1986, p. 798

8. Faddeev, L., Reshetikhin, N.Yu., Takhtajan, L.A.: Quantum groups. In: Yang, C.N., Ge, M.L. (eds.) Braid groups, knot theory and statistical mechanics. Singapore: World Scientific 1989

9. Faddeev, L.D., Takhtajan, L.A.: Liouville model on the lattice. Lect. Notes Phys. 246, 166 (1986)

10. Jimbo, M.: A $q$-difference analogue of $U(g)$ and the Yang-Baxter equation. Lett. Math. Phys. 10, 63 (1985)

Jimbo, M.: A $q$-analogue of $U(g l(N+1))$, Hecke algebra, and the Yang-Baxter equation. Lett. Math. Phys. 11, 247 (1986)

11. Kirillov, A.N., Reshetikhin, N.Yu.: $q$-Weyl group and a multiplicative formula for universal $R$-matrices. Commun. Math. Phys. 134, 421 (1990)

12. Klauder, J.R.: A coherent-state primer. In: Klauder, J.R., Skagerstam, B.-S. (eds.) Coherent states. Singapore: World Scientific 1985

13. Levendorskii, S., Soibel'man, Ya: Algebras of functions on compact quantum groups, Schubert cells and quantum tori. Commun. Math. Phys. 139, 141 (1991)

14. Lu, J.H., Weinstein, A.: Poisson Lie groups, dressing transformations and Bruhat decompositions. J. Diff. Geom. 31, 501 (1990)

15. Perelomov, A.M.: Generalized coherent states and their applications. Berlin, Heidelberg, New York: Springer 1986

16. Podlés, P.: Complex quantum groups and their real representations. RIMS preprint (1991)

17. Podlés, P., Woronowicz, S.L.: Quantum deformation of Lorentz group. Commun. Math. Phys. 130, 381 (1990)

18. Reshetikhin, N.Yu., Semenov-Tian-Shansky, M.A.: Quantum $R$-matrices and factorization problems. J. Geom. Phys. 5, 533, (1988)

19. Reshetikhin, N.Yu., Takhtajan, L.A., Faddeev, L.D.: Quantization of Lie groups and Lie algebras. Algebra i analiz 1, 178 (1989) (in Russian)

20. Semenov-Tian-Shansky, M.A.: Dressing transformation and Poisson Lie group actions. Publ. RIMS, Kyoto University 21, 1237 (1990)

21. Sheu, A.J.L.: Quantization of the Poisson $S U(2)$ and its Poisson homogeneous space - the 2-sphere. Commun. Math. Phys. 135, 217 (1991)

22. Soibel'man, Ya.S.: Algebra of functions on the compact quantum group and its representations. Algebra i analiz 2, N1 (1990) (in Russian)

23. Soibel'man, Ya.S.: Irreducible representations of the function algebra on the quantum group $S U(n)$, and Schubert cells. Soviet. Math. Dokl. 40, 34 (1990)

24. Takhtajan, L.A.: Introduction to quantum groups. Lect. Notes. Phys. 370, 3 (1990)

25. Tuynman, G.M.: Generalized Bergman kernels and geometric quantization. J. Math. Phys. 28, 574 (1987)

26. Tjerk Koelink, H.: On $*$-representations of the Hopf $*$-algebra associated with the quantum group $U_{q}(n)$. Preprint

27. Vaksman, L.L., Soibel'man, Ya.S.: On algebras of functions on the quantum group $S U(n+1)$ and on the odd-dimensional quantum spheres. Algebra i analiz 2, N5 (1990) (in Russian)

28. Vaksman, L.L., Soibel'man, Ya.S.: Function algebra on quantum group $S U(2)$. Funk. Analiz i Ego Priloz. 22, N3, 1 (1988) (in Russian)

29. Woodhouse, N.: Geometric quantization. Oxford: Clarendon Press 1980

30. Woronowicz, S.L.: Compact matrix pseudogroups. Commun. Math. Phys. 111, 613 (1987)

31. Woronowicz, S.L.: Twisted $S U(2)$ group. An example of a non-commutative differential calculus. Publ. RIMS, Kyoto University 23, 117 (1987) 